\title{
Modulation of the gut microbiota-adipose tissue-muscle interactions by prebiotics
}

\author{
Julie Rodriguez and Nathalie M Delzenne \\ Metabolism and Nutrition Research Group, Louvain Drug Research Institute, UCLouvain, Université catholique de Louvain, Brussels, Belgium \\ Correspondence should be addressed to N M Delzenne: nathalie.delzenne@uclouvain.be
}

\begin{abstract}
The gut microbiota is now widely recognized as an important factor contributing to the regulation of host metabolic functions. Numerous studies describe an imbalance in the gut microbial ecosystem in response to an energy-dense diet that drives the development of metabolic disorders. In this context, the manipulation of the gut microbiota by food components acting as prebiotics appears as a promising strategy. Several studies have already investigated the beneficial potency of prebiotics, mostly inulin-type fructans, on host metabolism and key intestinal functions including gut hormone release. For the last 20 years, several non-digestible compounds present in food have been shown to modulate the gut microbiota and influence host metabolism in essential organs involved in the control of energy homeostasis. To date, numerous reviews summarize the impact of prebiotics on the liver or the brain. Here we propose to describe the mechanisms by which prebiotics, through modulation of the gut microbiota and endocrine functions, modulates the metabolic cross-talk communication between the gut, the adipose tissue and skeletal muscles.
\end{abstract}

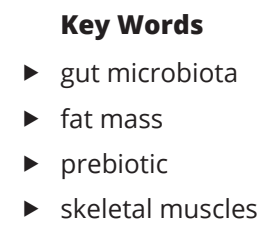

Journal of Endocrinology (2021) 249, R1-R23

\section{Glucose homeostasis disruptions are associated with changes in the gut microbiota in diabetes and obesity}

Obesity is often associated with a range of metabolic alterations including insulin resistance, type 2 diabetes, dyslipidemia, cardiovascular or non-alcoholic fatty liver diseases. Even if these alterations certainly result from a dysregulation of the balance between energy intake and energy expenditure, it has been now clearly established that some other factors can participate to the development and progression of cardio-metabolic diseases. Over the last decade, gut microbial changes (composition and/or function) have been associated with obesity, metabolic disorders, and 'pre' diabetes leading to a 'dysbiotic state' (Qin et al. 2012, Allin et al. 2018). This suggests that the gut microbiota can be involved (as a cause or consequence) in the insulin resistance process, and therefore in the disruption of endocrine system. Indeed, compared to non-diabetic individuals, the gut microbiota from type 2 diabetic patients (T2D) were characterized by a lower abundance of several bacteria (Clostridiales spp. SS3/4, Eubacterium rectale, Faecalibacterium prausnitzii, Roseburia intestinalis and Roseburia inulinivorans) able to generate butyrate, one important short chain fatty acid (SCFA) produced upon fermentation of carbohydrates and fibers (Qin et al. 2012). The decreased abundance of butyrate-producing bacteria was confirmed in a Chinese cohort and seemed to appear early, in the period of prediabetes (Zhang et al. 2013). Indeed, the abundance of some bacteria (such as Akkermansia muciniphila and Faecalibacterium prausnitizii) were higher in individuals with normal glucose tolerance, compared to prediabetic subjects (Zhang et al. 2013). 
Butyrate has been previously described as a beneficial metabolite for the intestinal physiology (including gut barrier function, intestinal inflammation, adherence of beneficial microbes in colonic epithelial cells, gut permeability) but it can also be linked with metabolic health by regulating body weight gain and glucose homeostasis (Blaak et al. 2020).

A higher abundance of opportunistic pathogens (Bacteroides caccae, Clostridium hathewayi, Clostridium ramosum, Clostridium symbiosum, Eggerthella lenta and Escherichia coli) characterizes diabetics from non-diabetic controls (Qin et al. 2012). Moreover, the fecal metagenome from European women with normal, impaired or diabetic glucose control revealed positive associations for Clostridium clostridioforme with triglycerides and C-peptide levels, and Lactobacillus gasseri with fasting glucose and hemoglobin A1c (HbA1c), in T2D patients (Karlsson et al. 2013). In addition, 21 metagenomic clusters were depleted in T2D women, particularly clusters belonging to Roseburia genus, Clostridium species, Eubacterium eligens, several Clostridiales and one Bacteroides intestinalis, the latter being negatively correlated with waist circumference and insulin level. In addition, among a population of Danish obese individuals, those with a low bacterial richness seem to be susceptible to have a more marked adiposity, insulin resistance and inflammatory phenotype compared to the ones with a higher bacterial richness (Le Chatelier et al. 2013). Individuals with reduced microbial gene richness were also more susceptible to present dysmetabolism and low-grade inflammation in a cohort of French obese or overweight subjects (Cotillard et al. 2013). Interestingly, a diet-induced weight loss improved the gut bacterial richness and partially reversed the metabolic alterations. More recently, in the same French cohort, the authors established a link between a low gut microbiota richness and a high level of ceramides, lipids mediators involved in the development of type 2 diabetes (Kayser et al. 2019). They highlighted a decreased abundance of Methanobreviibacter smithii and anti-inflammatory bifidobacteria species associated with higher ceramides levels. Another recent study highlighted that Danish adults with prediabetes exhibit an aberrant gut microbiota in which the main differences (compared to individuals with normal glucose regulation) are a lower abundance of both Clostridium genus and the mucindegrading bacterium A. muciniphila (Allin et al. 2018). However, the analysis of gut microbiota changes in the context of impaired glucose homeostasis is quite difficult since largely prescribed antidiabetic treatments, such as metformin, generate confounding effects by modulating per se the gut microbiota composition (Forslund et al. 2015, Rodriguez et al. 2018). In this context, a study performed in Swedish subjects naïve for diabetic treatments, showed that gut microbiota composition was altered in people with impaired glucose tolerance, glucose intolerance (impaired glucose tolerance associated to impaired fasting glucose) and T2D individuals, but not in those with impaired fasting glucose ( $\mathrm{Wu}$ et al. 2020). This study confirmed a decreased abundance of several butyrate producers, but also highlighted an alteration of butyrate production with glycemic status in both prediabetes and T2D groups. Interestingly, these gut microbiota alterations were present in prediabetic individuals, but increased with glucose intolerance and linked with insulin resistance. In addition to the SCFA production alterations, an elegant review recently summarized the modification of gut microbiota dysfunctions that are occurring in type 2 diabetes patients, namely an increased sugar-related membrane transport that promotes cellular glucose uptake, a higher transport of the branched-chain amino acids (BCAA) and a lowered metabolism of cofactors and vitamins (Fan \& Pedersen 2020). This strongly supports the association between the gut microbiota and the endocrine system, especially in the glucose homeostasis control.

\section{The gut microbiota as modulator of host metabolism disturbances}

\section{The causal role of the gut microbiota in the development of obesity and insulin resistance}

In 2004, a pioneering study highlighted that the gut microbiota can control the host metabolism by regulating fat storage in murine model (Backhed et al. 2004). Indeed, the colonization of germ-free (GF) mice with cecal microbiota from the conventionally raised mice was sufficient to promote the triglycerides storage in adipocytes and insulin resistance, despite a reduction of energy intake. This supported for the first time that the gut microbiota has a causal role in the occurrence of adiposity and glucose homeostasis. In addition, the same group reported that, contrary to conventionalized mice, GF mice are protected against the obesity induced by a high-fat/high-sugar (HF/HS) diet (Backhed et al. 2007). This protection was associated with a higher phosphorylation of AMP-activated protein kinase, and its downstream targets involved in fatty acid (FA) oxidation, in both skeletal muscles and liver from GF mice, compared to conventionalized ones. The suppression of intestinal 
expression angiopoietin-like protein 4 (Angpt14) in GF mice abolished the protection of mice against the dietinduced obesity and was associated with increased serum insulin levels. Interestingly, the deficiency of Angptl4 in GF fed a high-fat/high-sugar diet is associated with a decreased expression of markers involved in FA oxidation in skeletal muscles, independently of AMPK regulation. In 2010, another team confirmed the resistance of GF mice to the high-fat/high-sugar diet-induced obesity (Rabot et al. 2010). They showed that this protection was due to an improvement of insulin sensitivity and a reduced plasma level of proinflammatory cytokine tumor necrosis factor alpha (TNF-alpha) in GF mice fed with a HF/HS diet, compared to conventional mice. Indeed, the inflammatory process creates a linkage between the gut microbiota and insulin resistance. In 2007, it was demonstrated in mice that a HF diet generated endotoxemia, defined as an increase of plasma lipopolysaccharide (LPS), a bacterial component from the Gram-negative bacteria (Cani et al. 2007a). This endotoxemia was associated with changes in the gut microbiota. Among these changes, a lower abundance of Bifidobacterium spp. and E. rectaleClostridium coccoides group were found. Interestingly, mimicking endotoxemia by infusing LPS induced body weight gain, adiposity, liver insulin resistance and triggered the expression of inflammatory markers in adipose tissue, liver and skeletal muscle. In addition, antibiotic treatment in both high-fat-fed and $o b / o b$ mice reduced endotoxemia, glucose intolerance, fat mass expansion and inflammation in adipose tissue. Finally, colonization of GF Swiss Webster mice with a microbiota obtained from conventionally raised mice has been shown to promote inflammation in the white adipose tissue (WAT) by favoring the recruitment of macrophages, LPS being involved in this process (Caesar et al. 2012). In addition to the involvement of gut microbiota, the type of dietary lipids in the diet also plays a crucial role in the development of WAT inflammation. Indeed, mice fed lard for 11 weeks exhibit WAT inflammation, increased Toll-like receptor (TLR) activation and reduced insulin sensitivity compared with mice fed fish oil (Caesar et al. 2015). Actually, this result was associated with a different regulation of gut microbiota composition in response to different dietary fat sources. For instance, lard induced an increase of Bilophila, Bacteroides, Turicibacter genera whereas fish-oil favored the growth of bacteria associated with beneficial effects on health such as Akkermansia, Bifidobacterium or Lactobacillus genera (Caesar et al. 2015).

The causal role of the gut microbiota in insulin sensitivity has also been reported supported by fecal material transfer (FMT) interventions in humans (Vrieze et al. 2012). By infusing the intestinal microbiota from lean donors to individuals with metabolic syndrome, an improvement of insulin sensitivity and an increased abundance of butyrate-producing bacteria was observed in recipient patients after 6 weeks. Five years later, the same group repeated the intervention on other patients and demonstrated that insulin sensitivity improvement was accompanied by changes in plasma metabolites profiling (including modifications of $\gamma$-aminobutyric or lysophosphatidic acid) but no change in fasting plasma SCFA was reported (Kootte et al. 2017). Interestingly, the authors demonstrated that the baseline fecal microbiota composition has a determinant role in the improvement of peripheral insulin sensitivity since its composition can predict the success of the allogenic FMT. Indeed, metabolic responders were characterized by a lower microbial diversity, a higher abundance of Subdoligranulum variabile and Dorea longicatena and lower abundance of Eubacterium ventriosum and Ruminococcus torques, compared to non-responders. Finally, recent works highlighted the importance for identifying and studying the impact of gut bacterial metabolites on health. Indeed, current research in the field of metabolic diseases focus on the relation of some bacterial products derived from carbohydrates or protein fermentation and the obesity-associated insulin resistance (Canfora et al. 2019).

\section{Focus on bacteria prone to improve glycemic control and metabolic disturbances}

In addition to the beneficial effects observed by transferring the fecal gut microbiota from lean donors into recipients with metabolic syndrome, it has been demonstrated that some specific bacteria can also improve the obesity and associated disorders, these bacteria being thus proposed as probiotics. Probiotics have been defined as 'live microorganisms that, when administered in adequate amounts, confer a health benefit on the host' (Hill et al. 2014). For instance, the administration of Bifidobacterium and Lactobacillus strains in HF-fed mice lowered the body weight gain, and improved glycemic control (Wang et al. 2015, Alard et al. 2016). Lactobacillus acidophilus NS1 (LNS1) inhibited the development of insulin resistance in HF mice by restoring the phosphorylation of Akt protein (involved in the insulin pathway) in the liver, adipose tissues and skeletal muscles (Park et al. 2018). Similarly, a treatment with a mix of probiotics containing Lactobacillus and Bifidobacterium strains also improved blood glucose in mice by regulating the phosphorylation of Akt protein 
both in the liver and skeletal muscles (Bagarolli et al. 2017). The treatment with $A$. muciniphila in HF-fed mice also reduced fasting glycemia and improved glucose tolerance (Everard et al. 2013, Plovier et al. 2017). This bacterium reduced the macrophage infiltration and improved the FA oxidation in visceral fat depots. Interestingly, the administration of pasteurized form of A. muciniphila enhances energy expenditure, oxygen consumption and physical activity in HF mice (Depommier et al. 2020). A. muciniphila reduces fat mass accumulation and decreases the expression of perilipin $2-a$ factor associated with lipid droplets - in both brown and white adipose tissues. Finally, in small cohort of obese human volunteers, three months of supplementation with pasteurized A. muciniphila improved insulin sensitivity, reduced insulinemia and plasma total cholesterol (Depommier et al. 2019). Other bacteria seem interesting in the context of insulin resistance such as Eubacterium hallii (now called Anaerobutyricum hallii). Daily oral gavage with A. hallii during 4 weeks significantly improved adiposity, insulin sensitivity and increased energy expenditure in $d b / d b$ mice (Udayappan et al. 2016). Additionally, oral treatment with F. prausnitzii in HF-fed mice improved hepatic fat content, both insulin sensitivity and inflammation in adipose tissues and increased skeletal muscle mass (Munukka et al. 2017). All these studies support the idea that specific bacteria isolated from the human gut microbiota participate to the regulation of host metabolism in peripheral tissues, targeting the liver, the adipose tissue and the muscle.

Finally, it is now obvious that the gut microbiota contributes to metabolic function in healthy individuals and disruption of this ecosystem promoted a diseased state associated with some detrimental metabolic outcomes: systemic inflammation, altered gut barrier function, insulin resistance, increased adiposity and plasma cholesterol or triglycerides... (Green et al. 2020). To date, there are different ways by which the gut microbiota can modulate the mechanisms of obesity including the processes of energy extraction and absorption, the induction or prevention of metabolic endotoxemia, and the production of bacterial metabolites such as SCFA which are able to stimulate the release of the satietyinducing hormones peptide YY (PYY) and glucagon-like peptide-1 (GLP-1) (da Silva et al. 2020). In this context, the modulation of gut microbiota by promoting the growth of some specific bacteria by a prebiotic approach can be an effective strategy to reverse some features of the microbial dysbiosis and improve some metabolic alterations. A recent review summarized the experimental work and the available human intervention studies that evaluated the anti-obesity effect of prebiotics (Cerdo et al. 2019). In our review, we describe the influence of prebiotic interventions on the gut microbiota composition and function, with a focus on the endocrine system regulation and consequences in the metabolism of the adipose tissues and skeletal muscles.

\section{Prebiotic approach in the modulation of the gut microbiota in obesity: gut endocrine system, adipose tissue and muscles as host targets}

Over the past decade, numerous studies have investigated the beneficial impact of gut microbiota modulation by prebiotics in the context of obesity and metabolic disorders. Prebiotics are defined as a 'substrates that are selectively utilized by host microorganisms conferring a health benefit' (Gibson et al. 2017). Indeed, several nondigestible carbohydrates can be fermented by the gut microbiota, including oligosaccharides, arabinoxylans, glucans, resistant dextrin or resistant starch. Table 1 summarizes the main studies performed in vivo in rodents supplemented with prebiotics. The outcomes reported relate to the gut microbiota composition (Fig. 1), and of biological markers of metabolic alterations.

From those data, we can elaborate the molecular mechanisms evoked to explain how prebiotics control energy homeostasis in obesity, which are summarized in Fig. 2. Due to the high number of reviews focusing on the impact of prebiotics on the liver steatosis and related metabolic disorders, we chose to present the existing literature related to the effects on the adipose tissue and the skeletal muscles, two organs playing a key role in storage vs oxidation of fatty acids. The modulation of the endocrine function by prebiotics is often cited as an important mechanism behind the systemic effects on lipid and glucose homeostasis, as well as on food related behavior.

In 2007, our team demonstrated that dietary supplementation with oligofructose (OFS) - a short chain inulin-type fructan - in HF-fed mice restored the amount of Bifidobacteria in the cecal content, normalized the endotoxemia and reduced fat mass accumulation (Cani et al. 2007b). This was linked to an improvement of glucose homeostasis through a better glucose-induced insulin secretion. Interestingly, OFS supplementation in $o b / o b$ or high fat fed mice reduced adipose tissue weights, increased muscle mass and regulated both pancreatic 


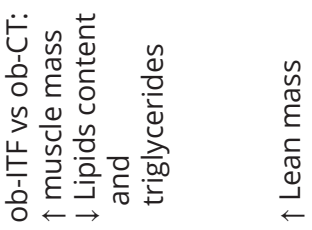

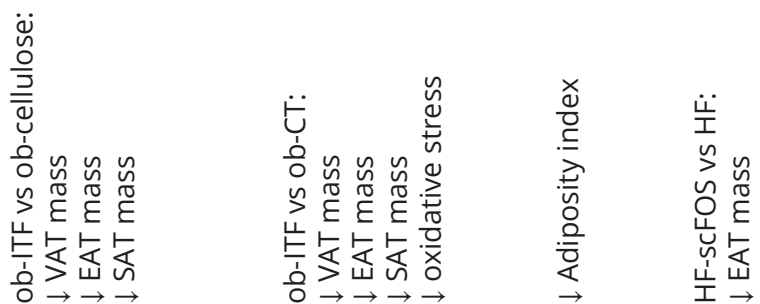

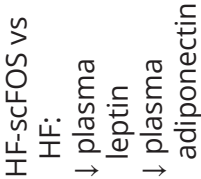

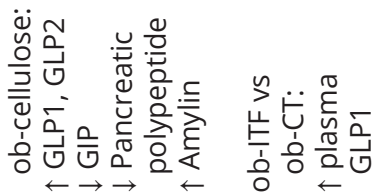

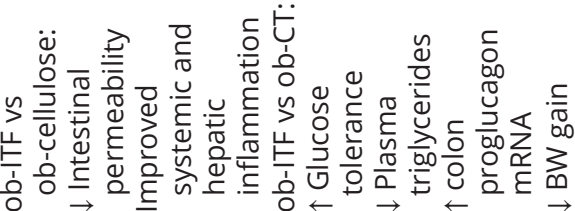

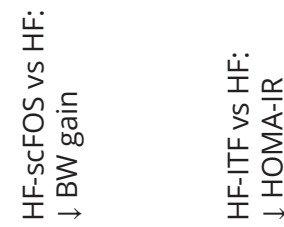
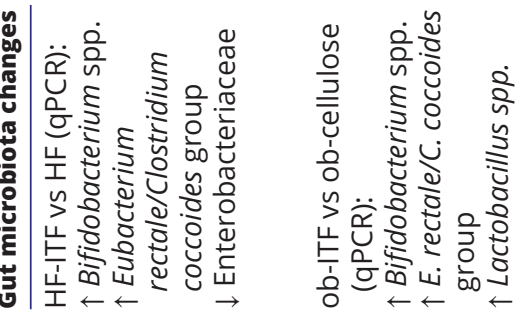

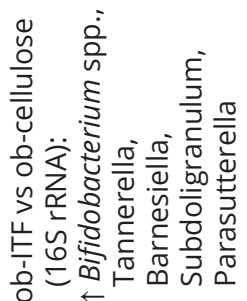
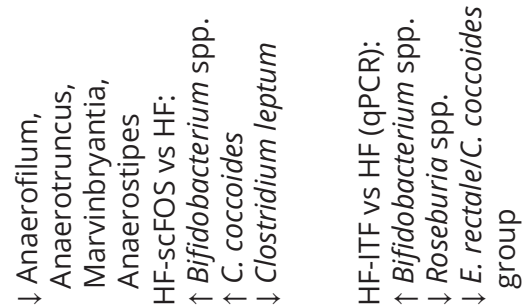

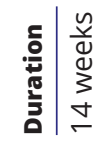

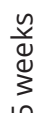

in

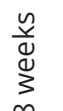

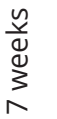
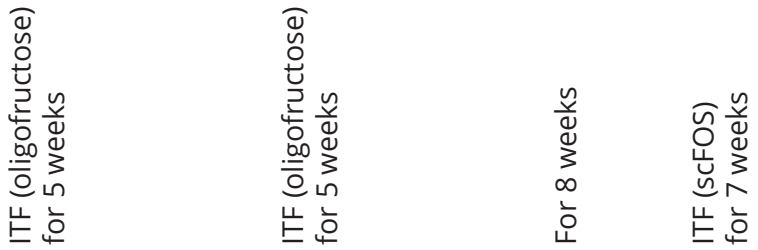

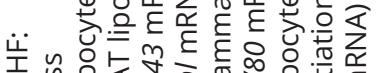
工

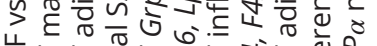

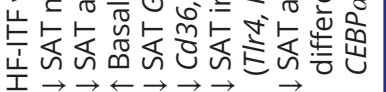

$$
\text { 亮 }
$$



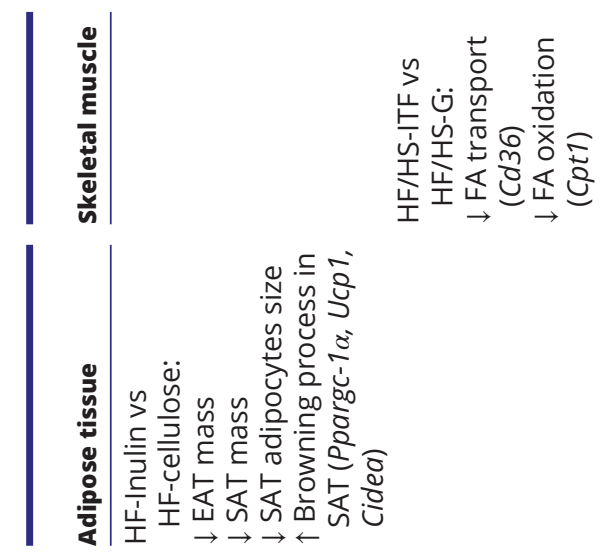

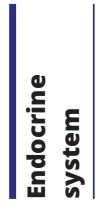
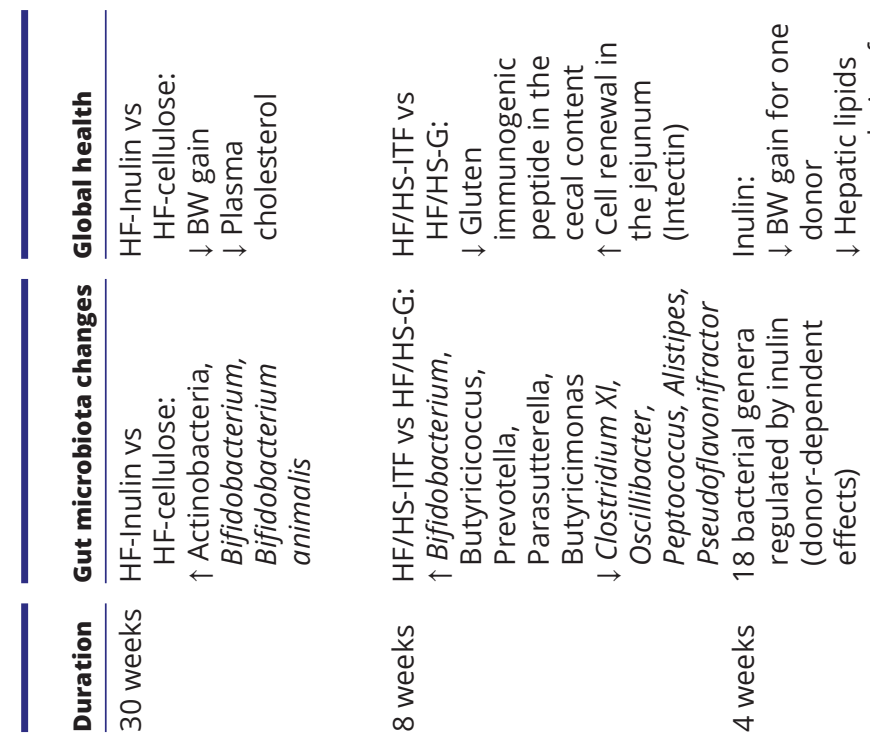

v
$\stackrel{v}{u}$
$\stackrel{u}{u}$
$\infty$
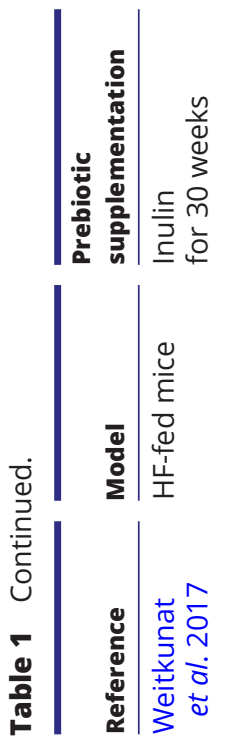

https://joe.bioscientifica.com
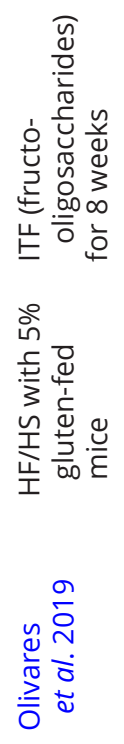
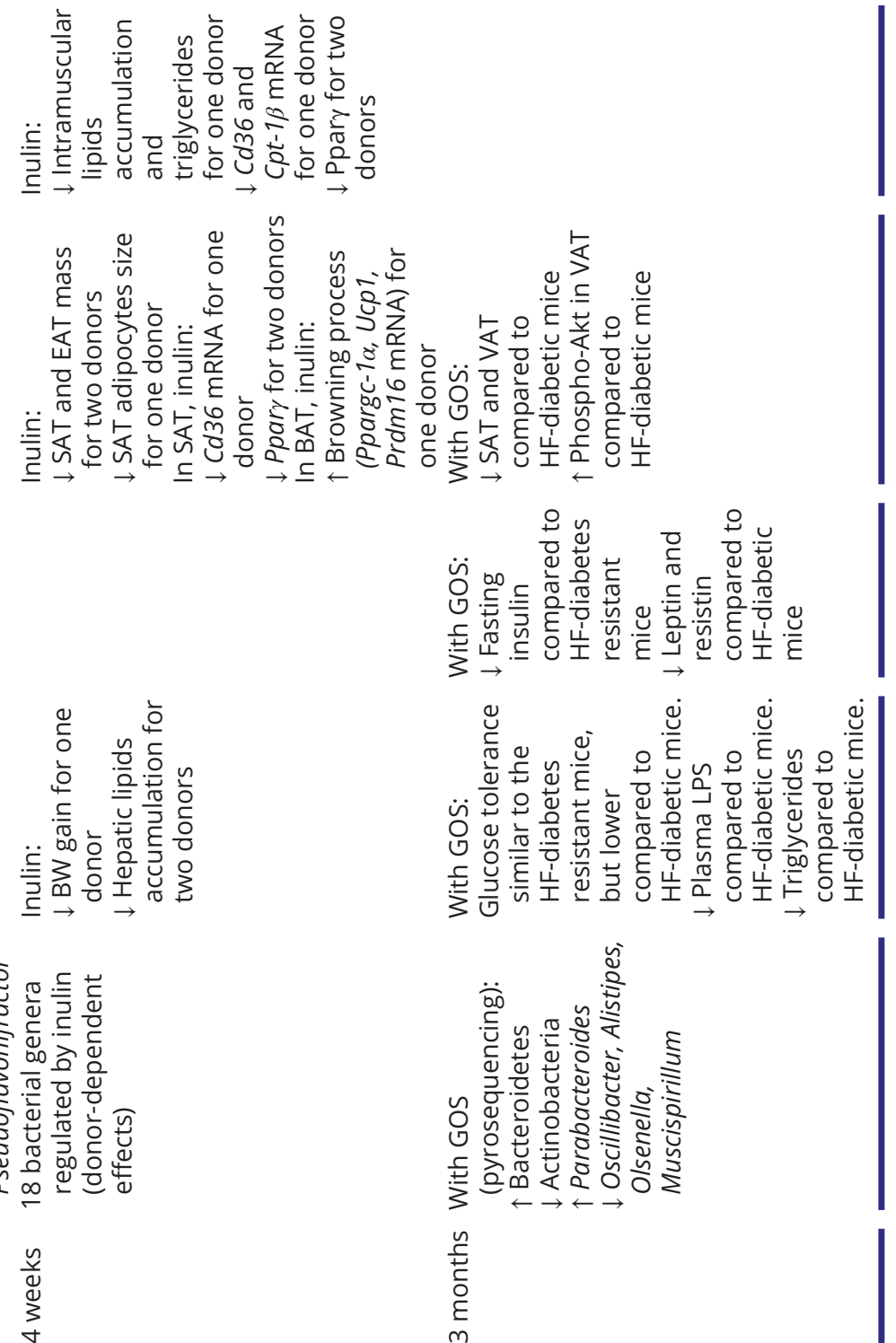

돋

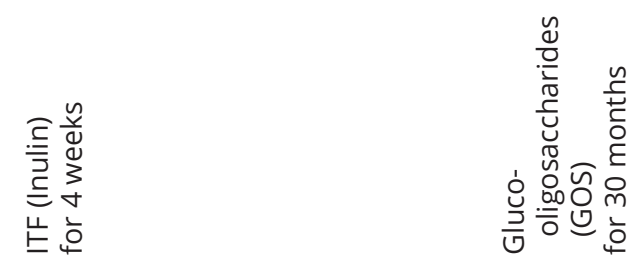

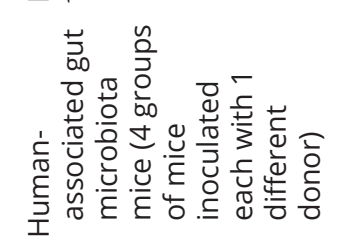
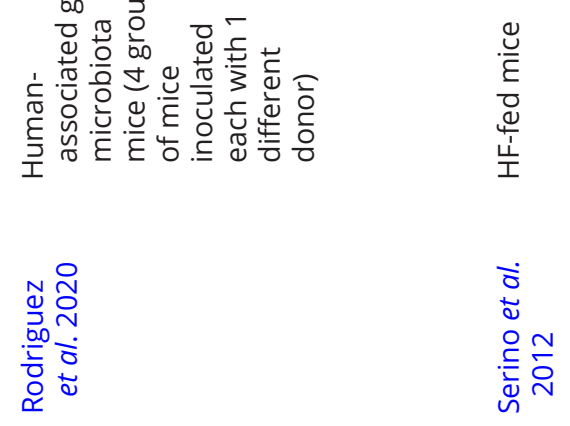

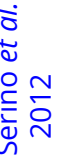


II

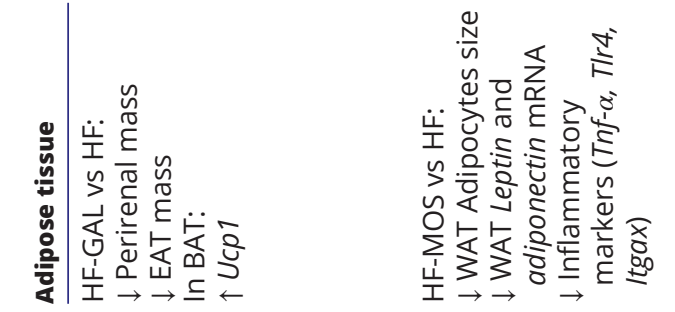

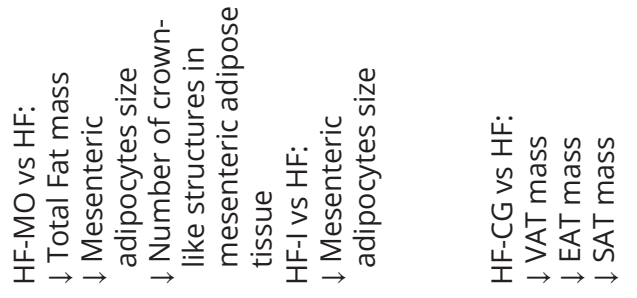

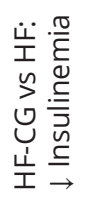

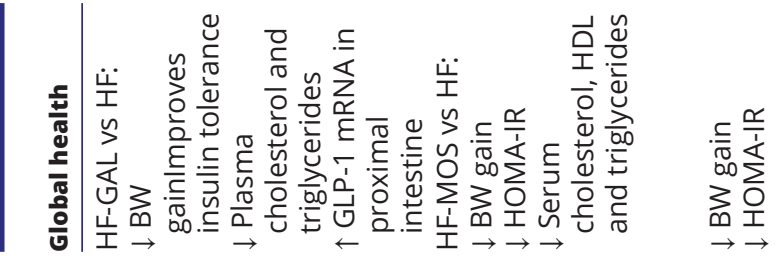

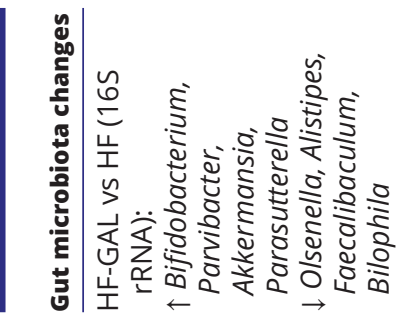

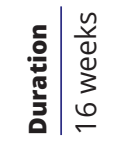

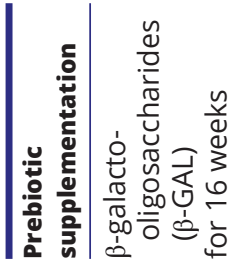

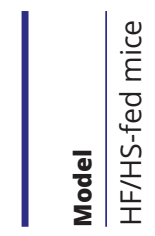

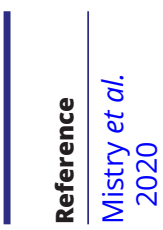

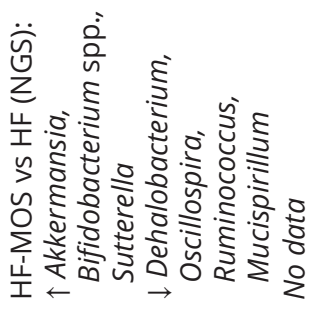

$\frac{n}{d}$
$\frac{1}{5}$
$\square$

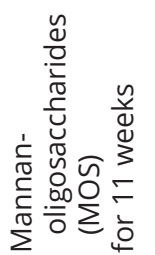

$\frac{\mathscr{U}}{\varepsilon}$
$\frac{0}{d}$
$\frac{\mathbb{4}}{1}$
$\frac{1}{I}$

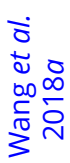

\section{$\frac{10}{8}$}
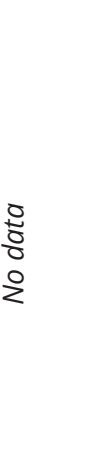

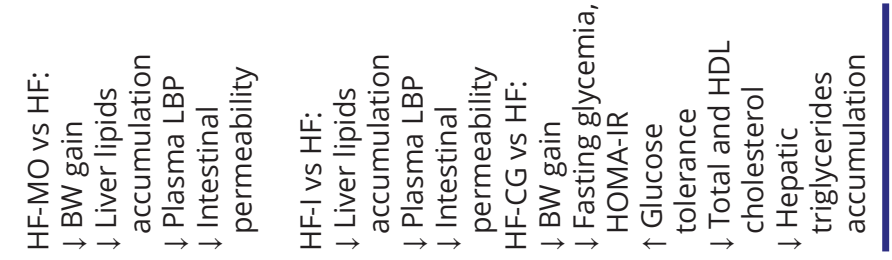

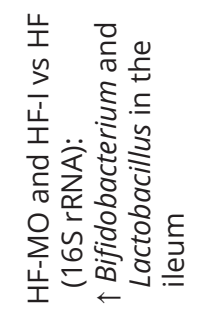

$\frac{n}{0}$

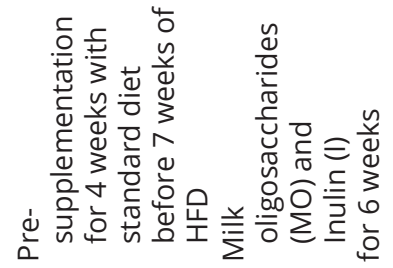

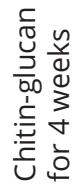

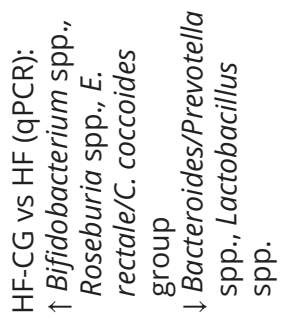

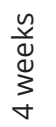

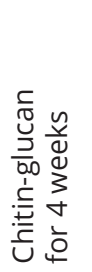

蒫

$\dot{\bar{\alpha}}$

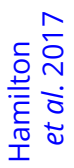



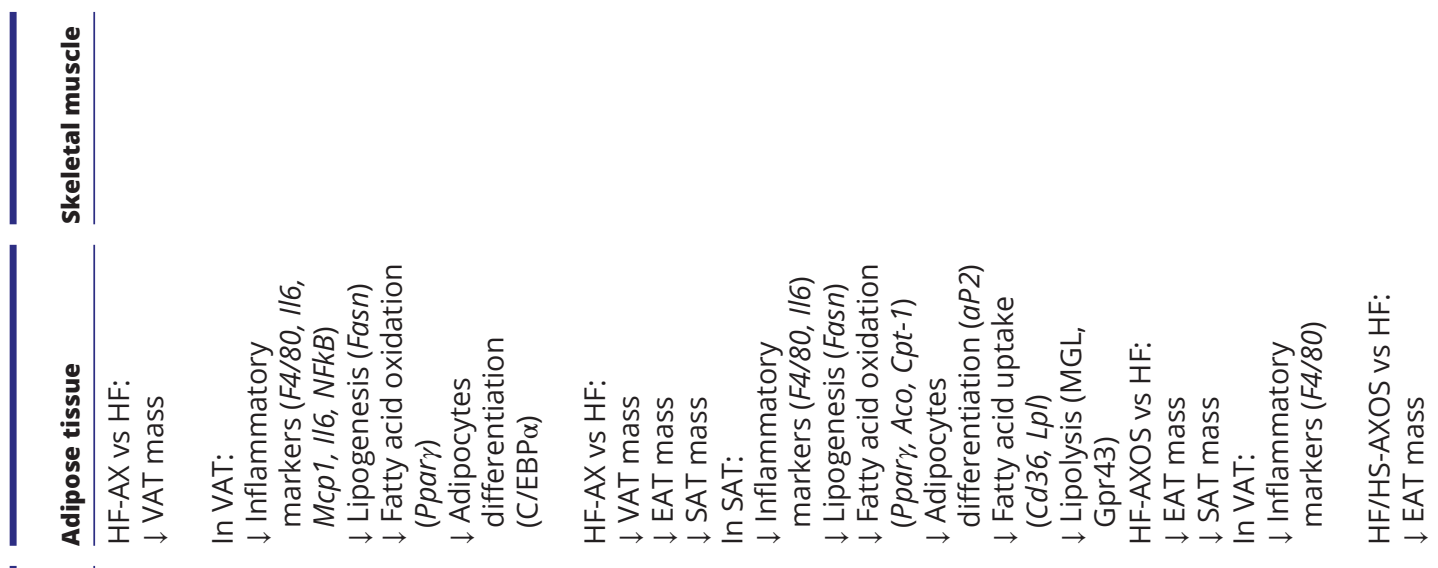

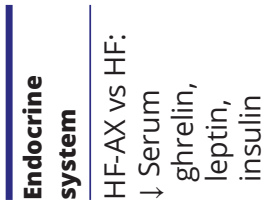

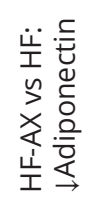

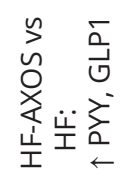
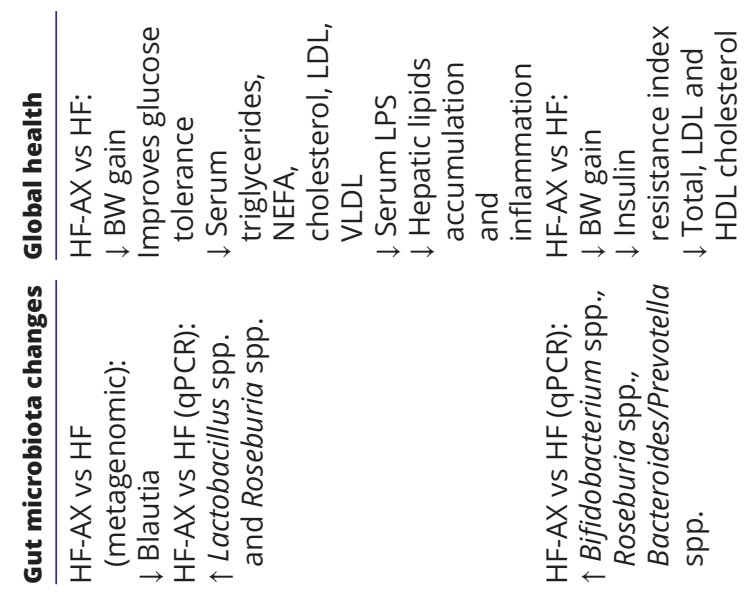

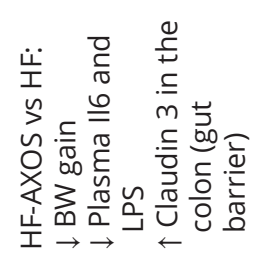
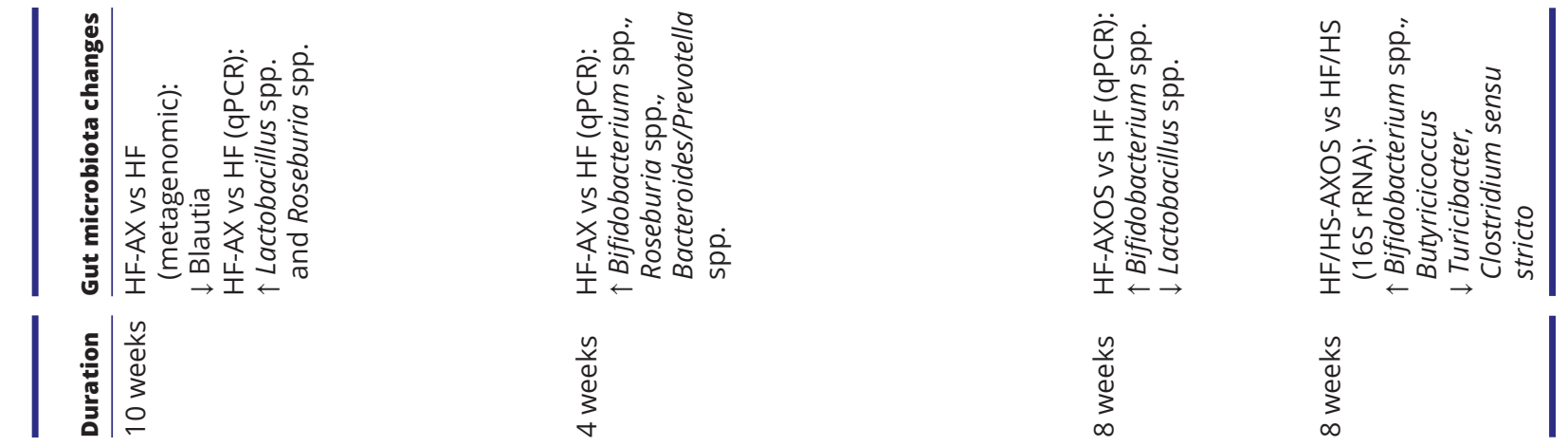

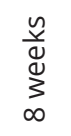

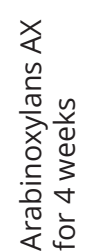

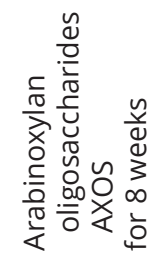

$$
\text { 峁 }
$$
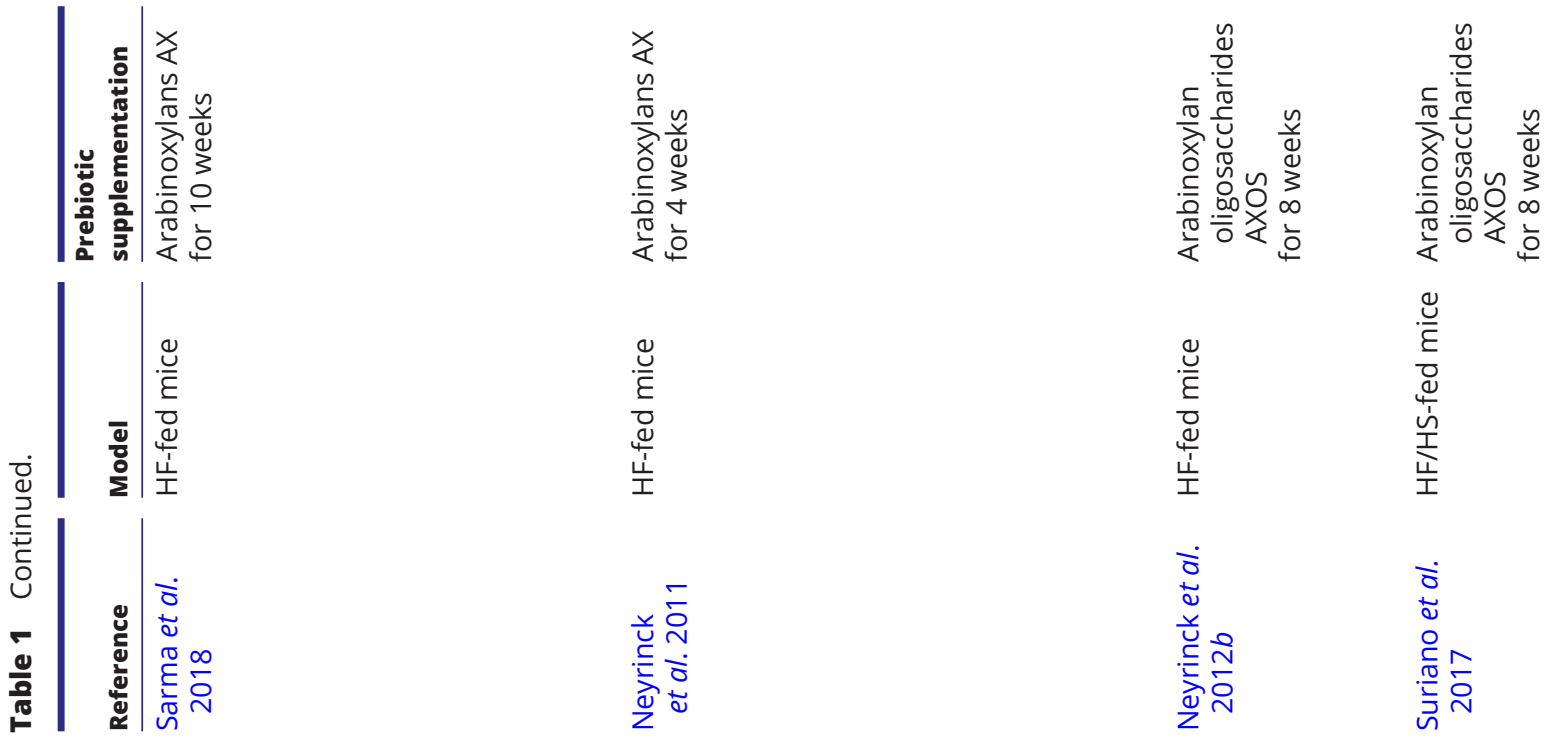

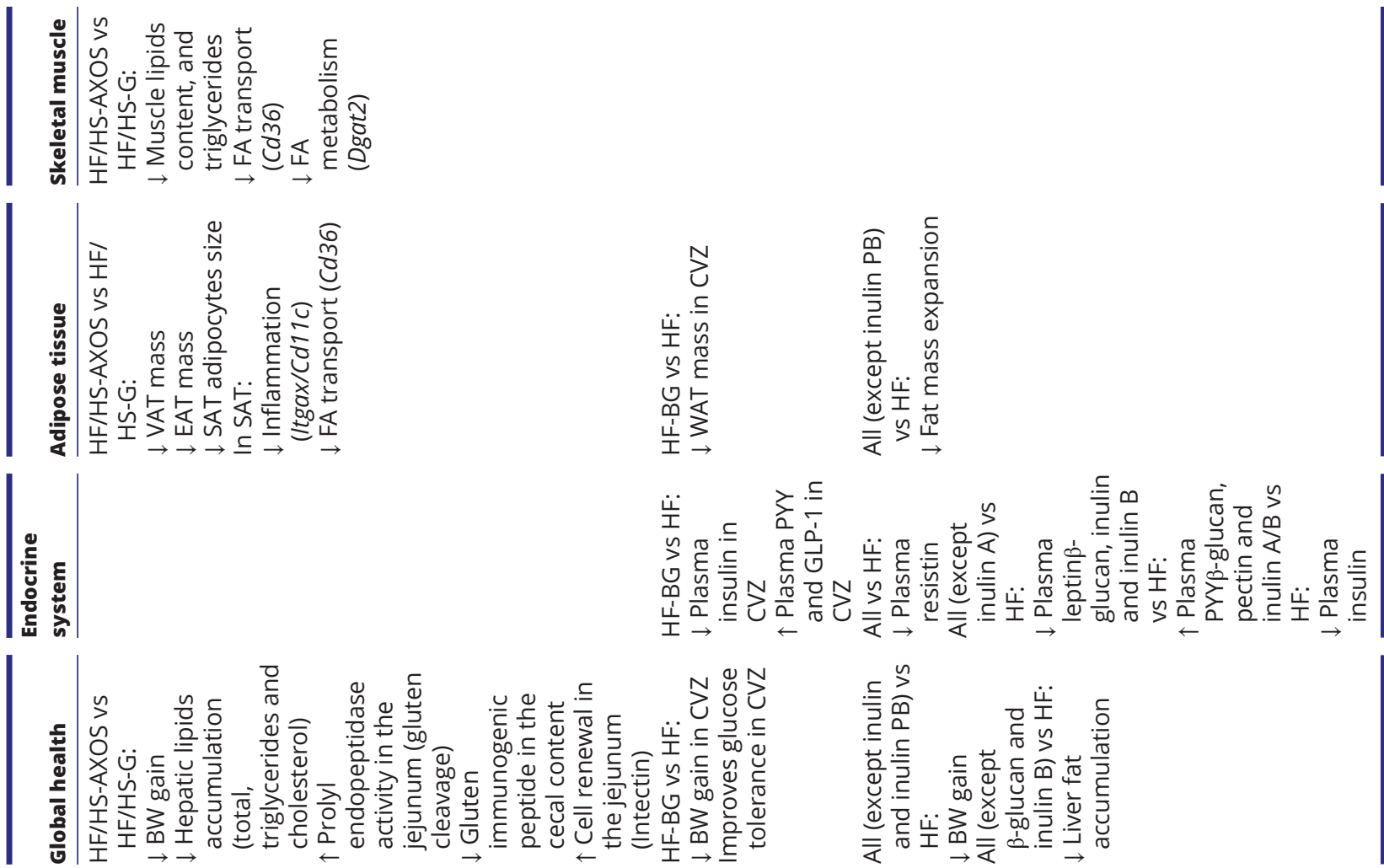

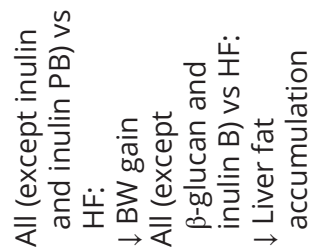
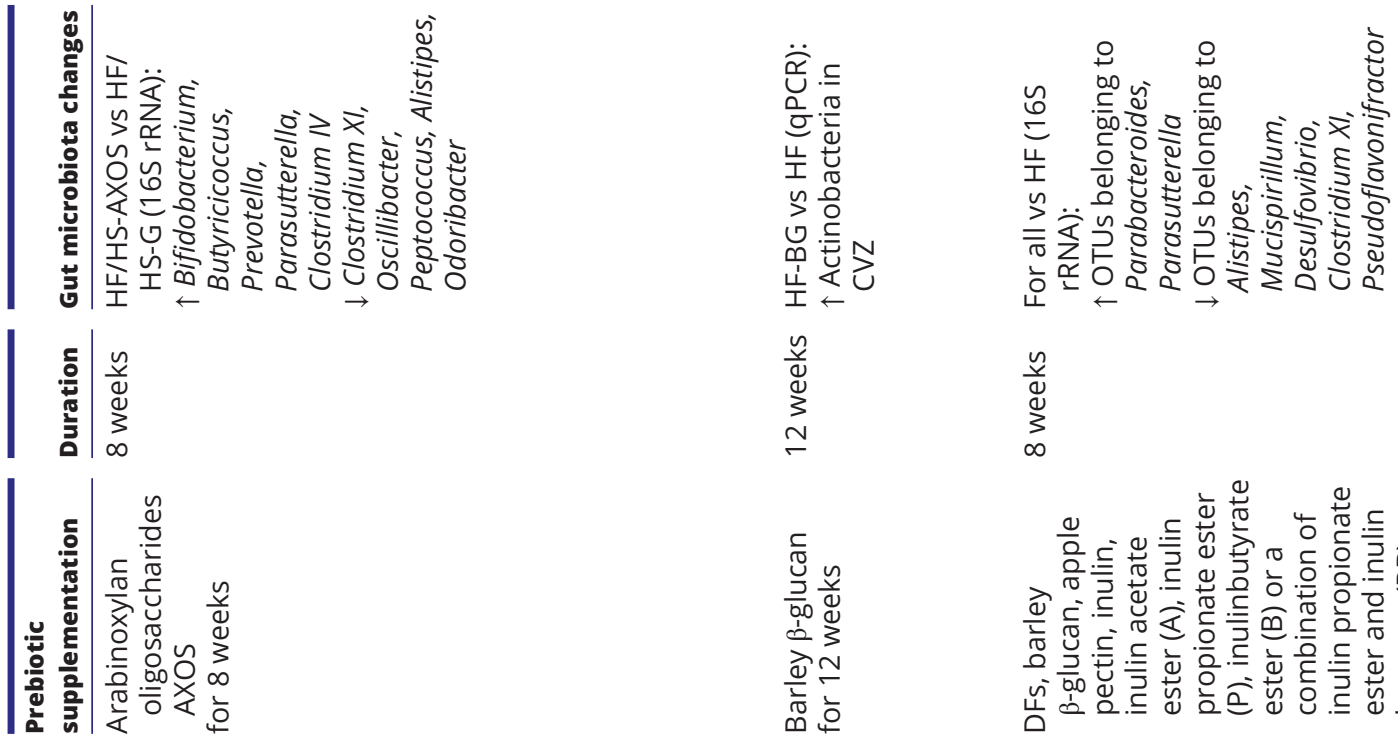

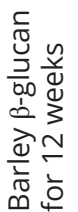
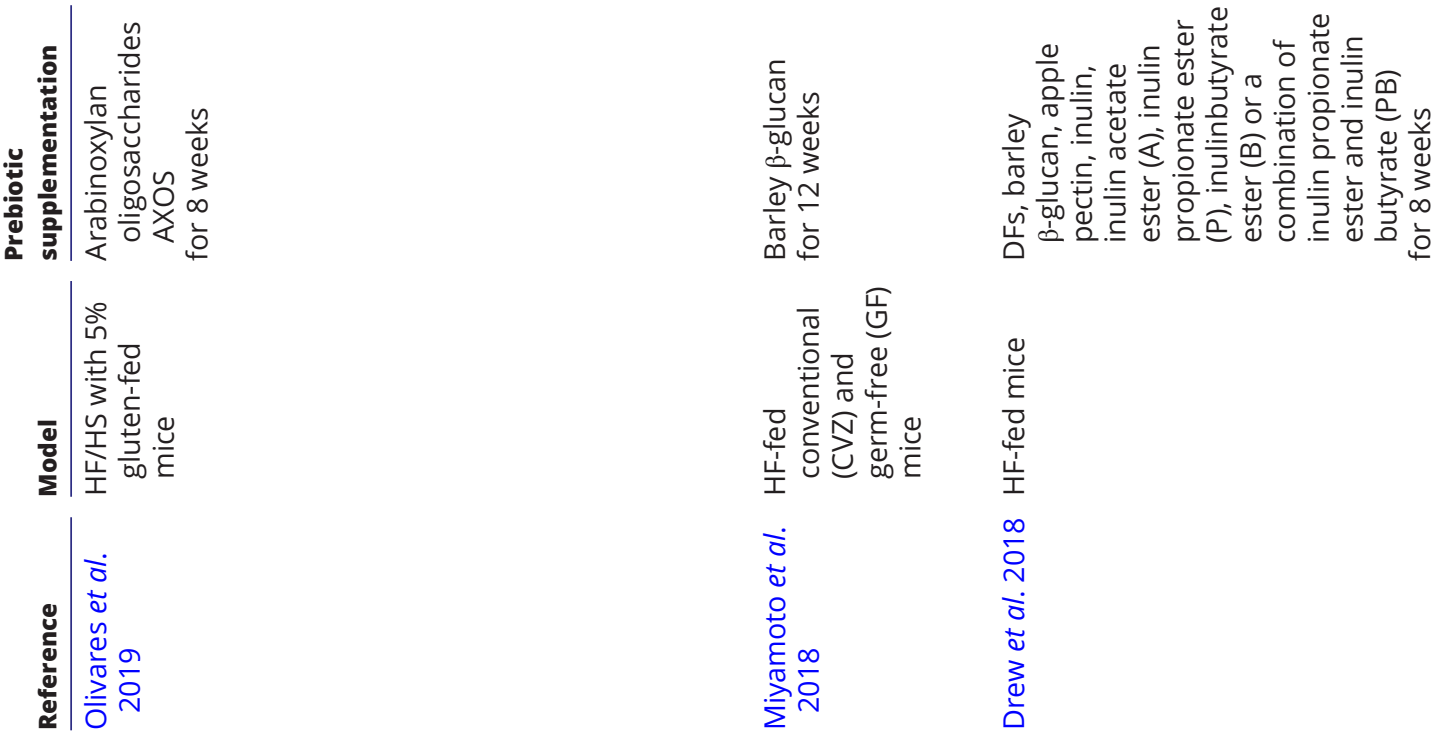<smiles>C1CCCCC1</smiles> 

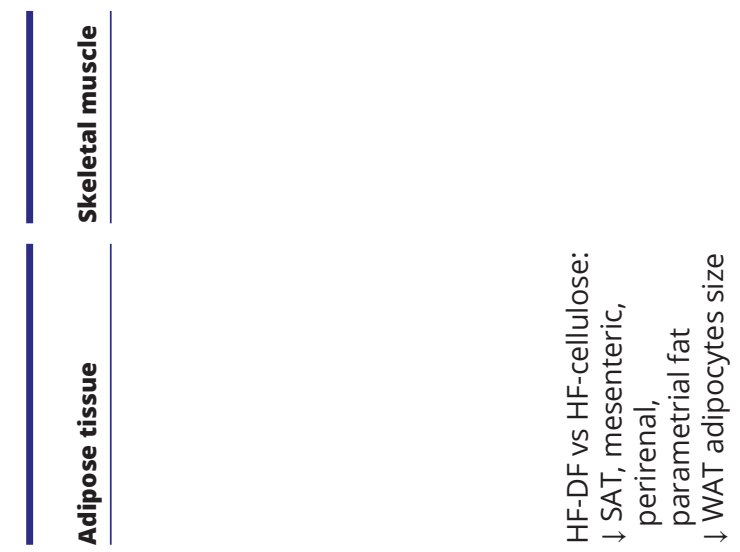

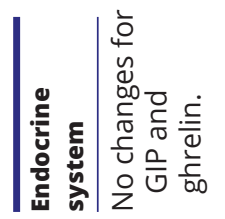
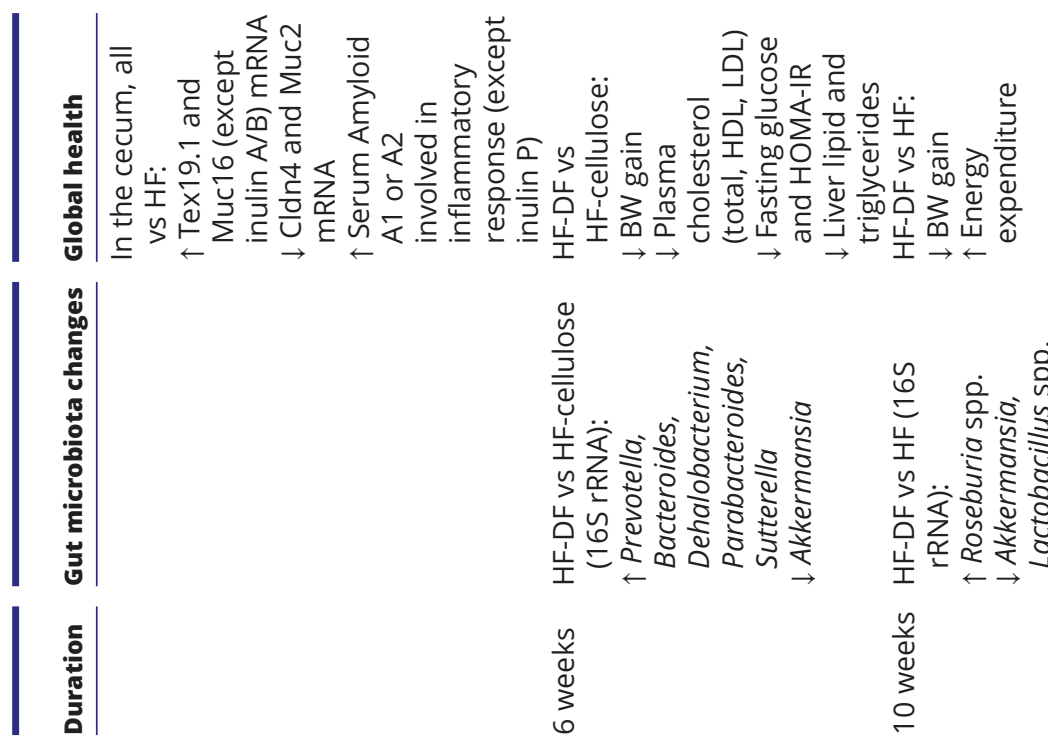

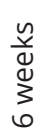

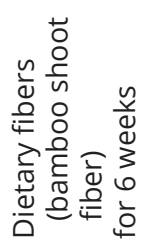

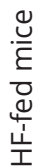

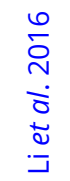

(C) 2021 Society for Endocrinology Published by Bioscientifica Ltd.
Printed in Great Britain
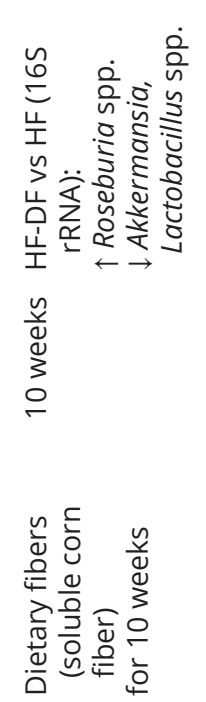

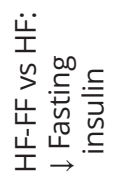
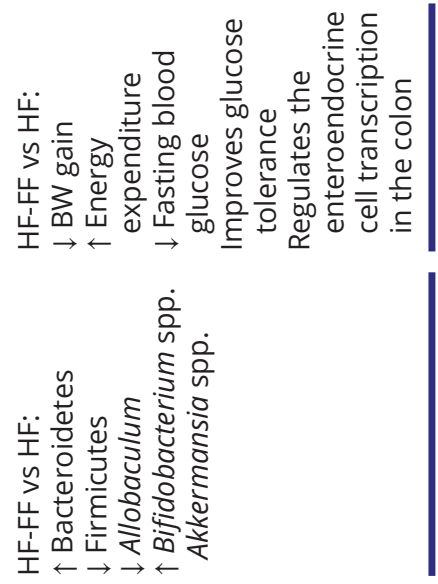

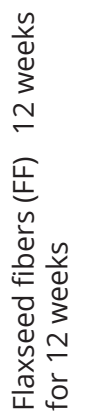
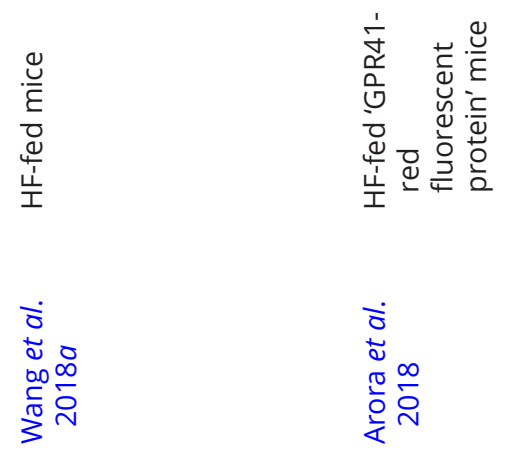


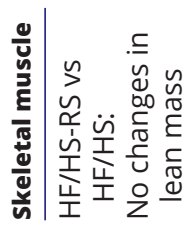

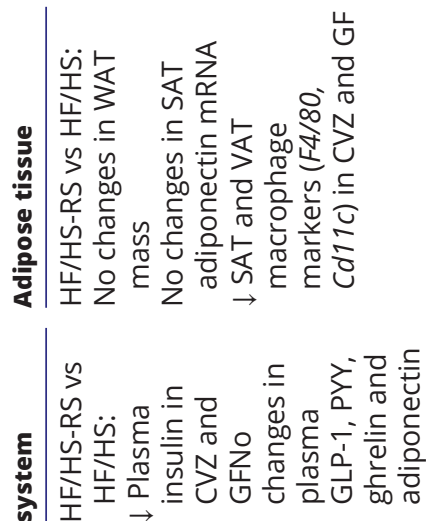

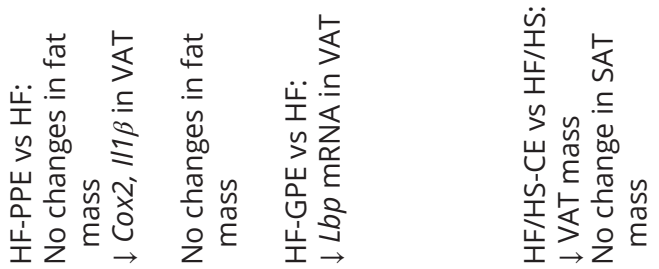

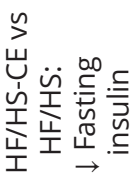

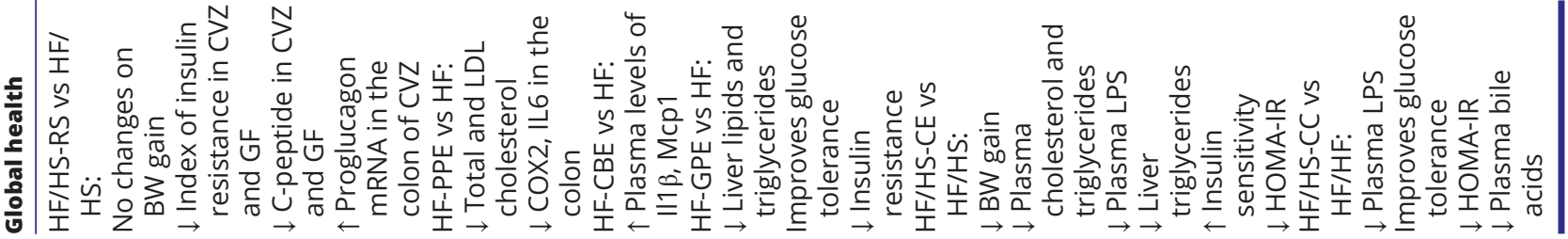
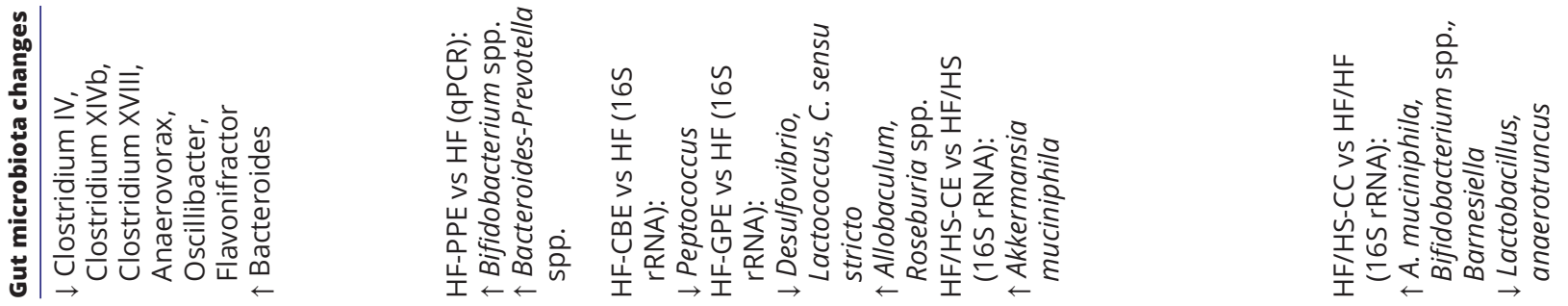

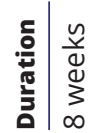

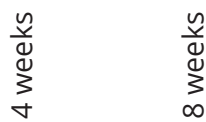

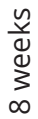

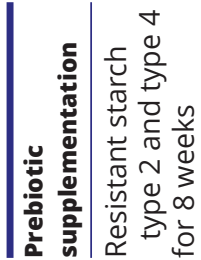

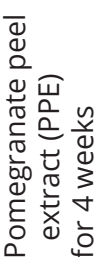

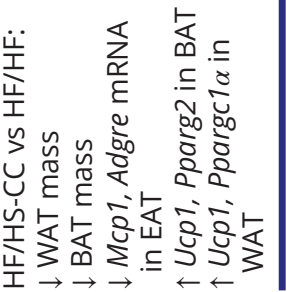

象

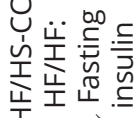
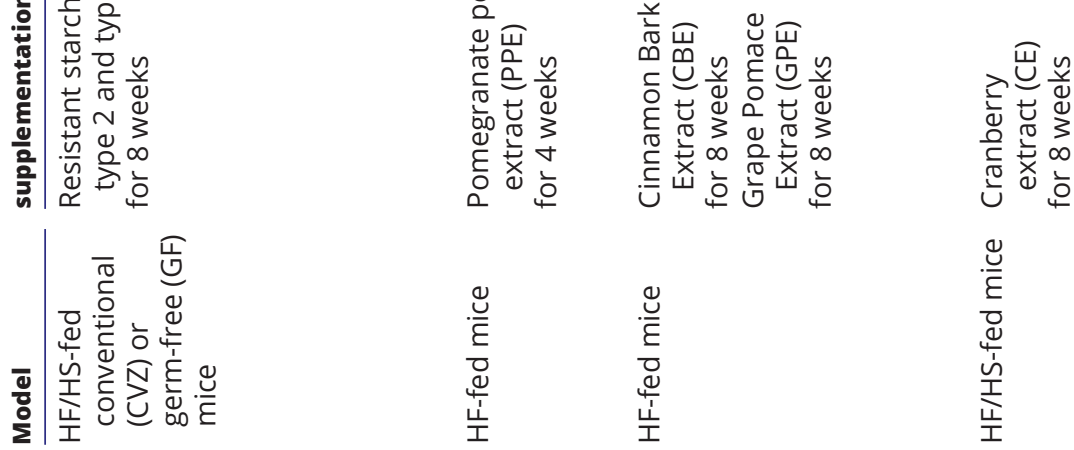

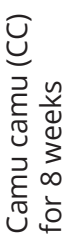

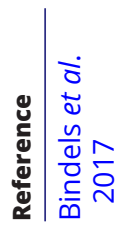

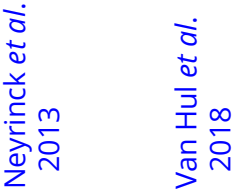

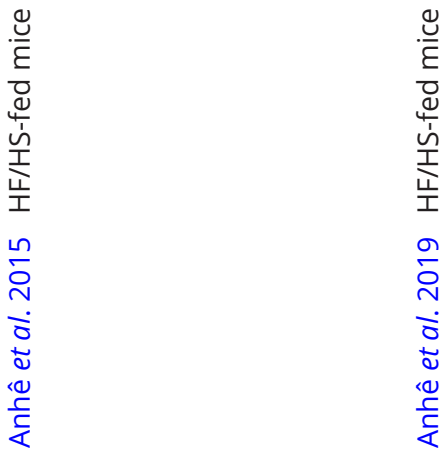




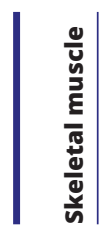

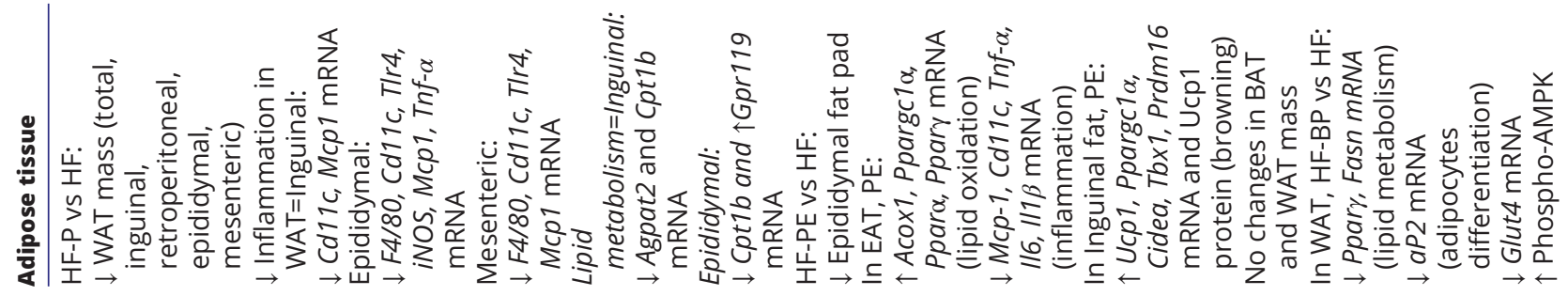
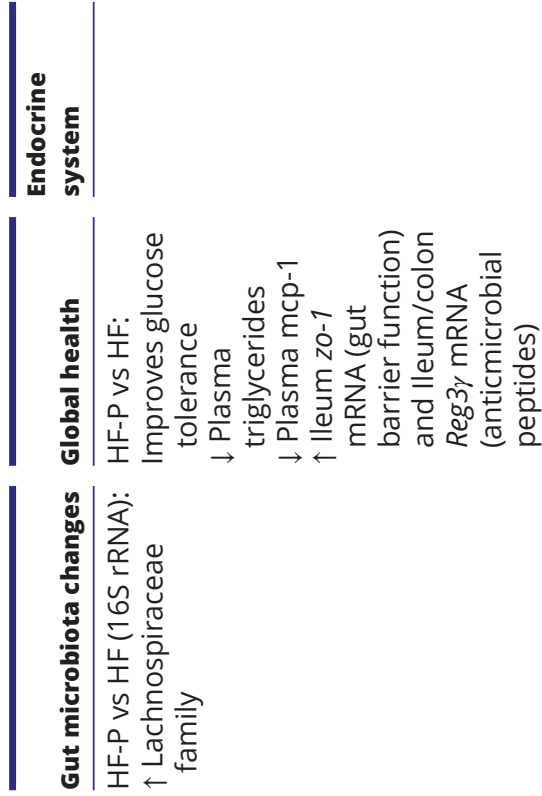

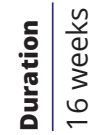
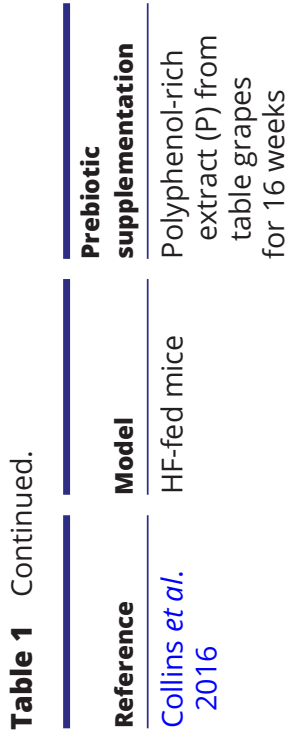

https://joe.bioscientifica.com https://doi.org/10.1530/JOE-20-0499

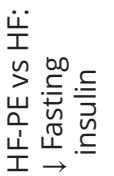
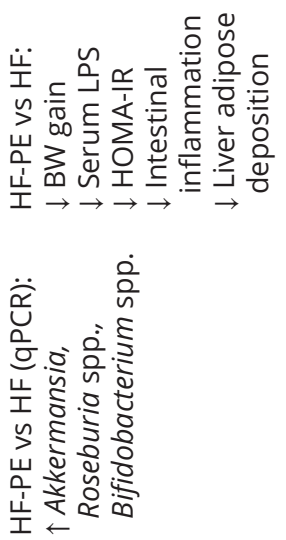

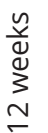

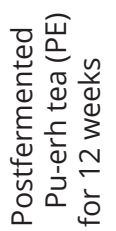

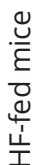

$\stackrel{\infty}{\circ}$

产

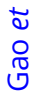

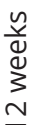

$\frac{\cup}{E}$
$\frac{\Theta}{0}$
$\stackrel{4}{4}$
$\frac{1}{I}$
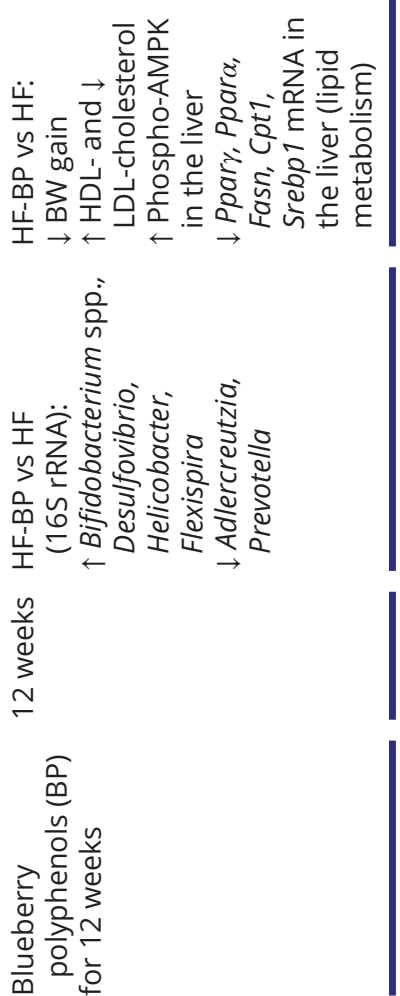

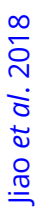




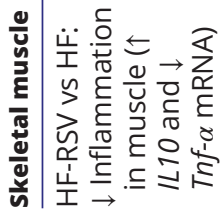

产

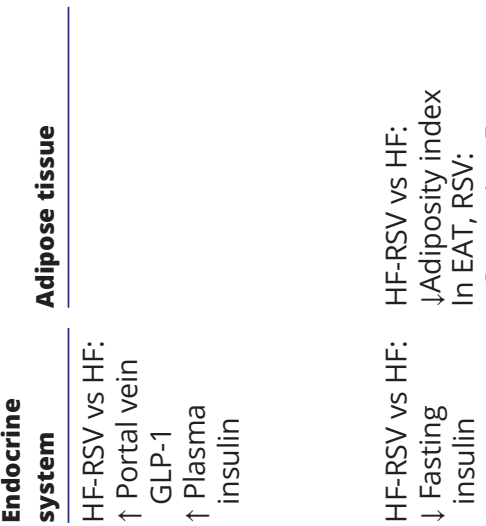

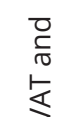

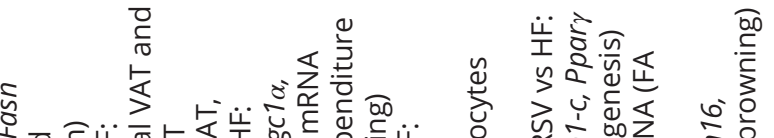

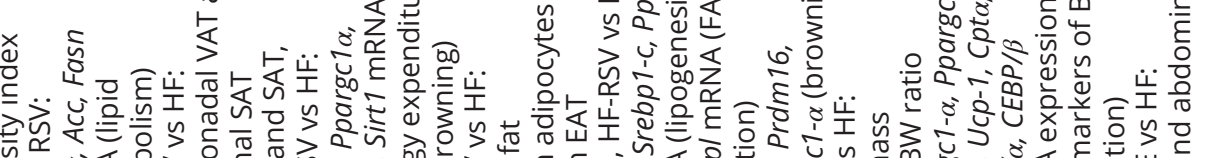

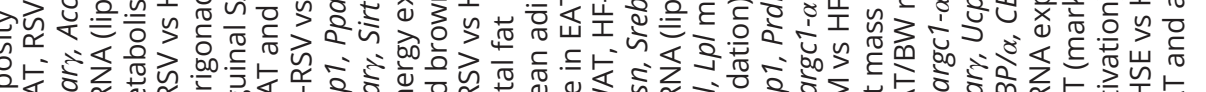

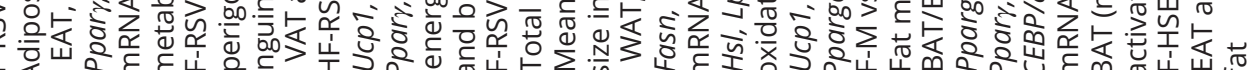
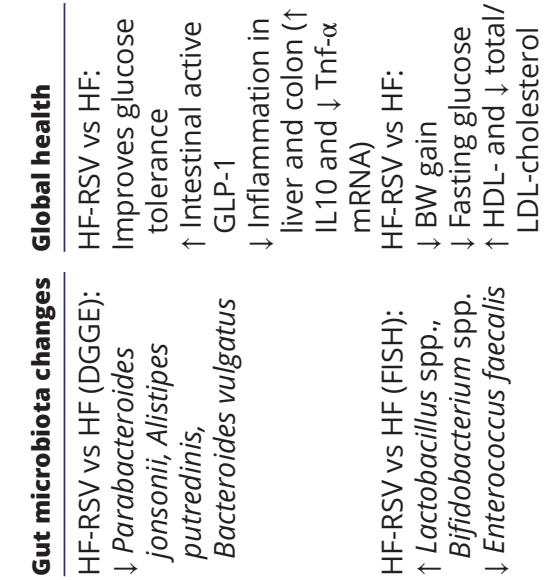

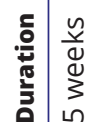

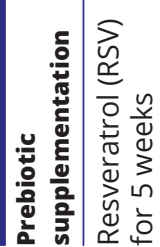

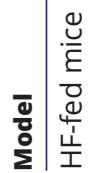

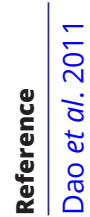

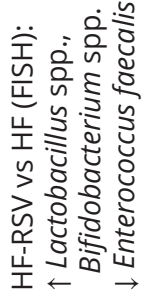

$\frac{n}{d}$

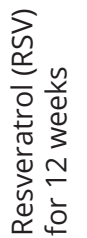

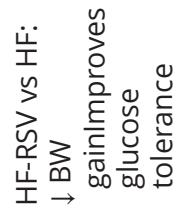

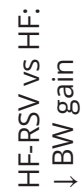

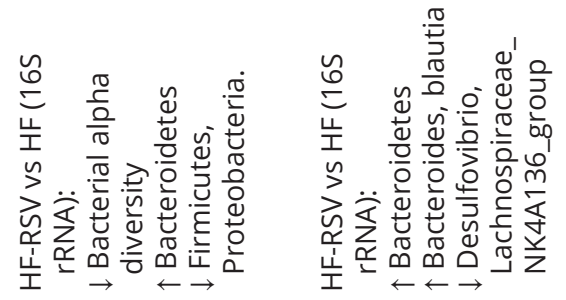

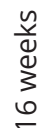
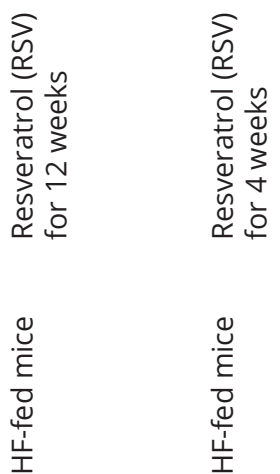

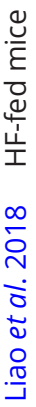

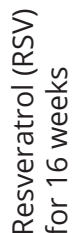

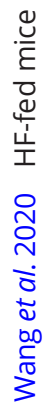

C 2021 Society for Endocrinology Published by Bioscientifica Ltd.

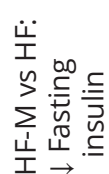

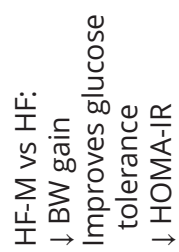

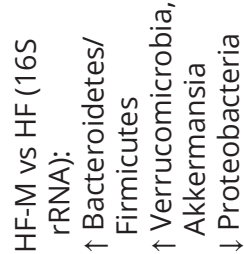

$\sum_{m}^{\stackrel{n}{\dddot{d}}}$
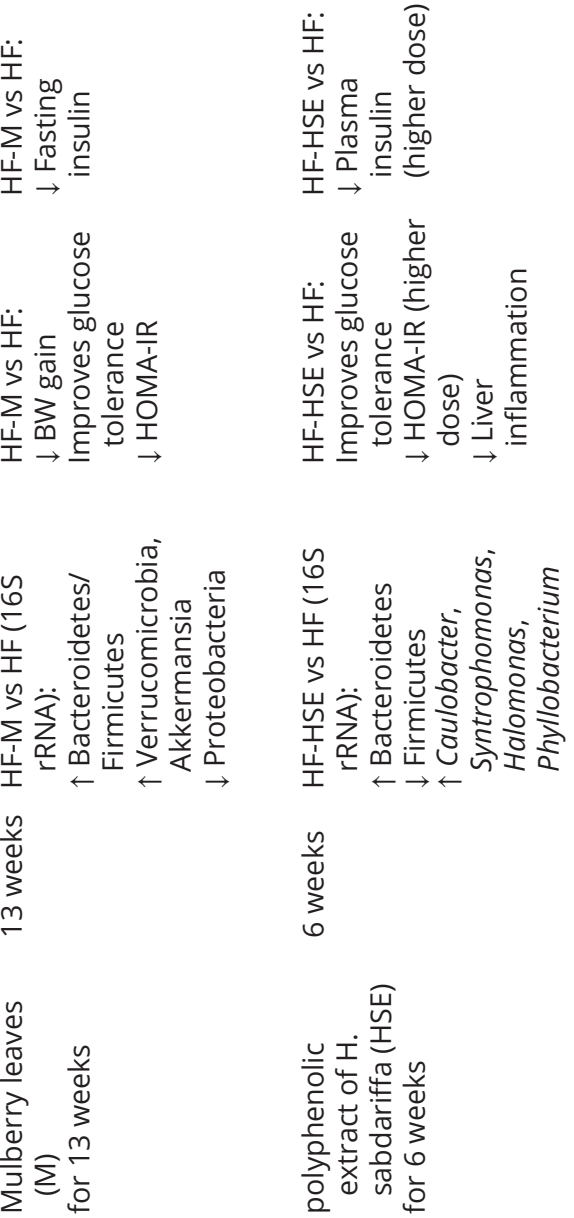

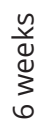
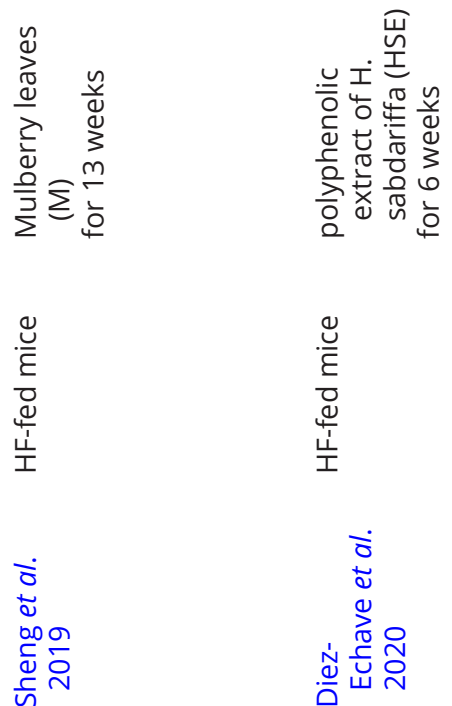

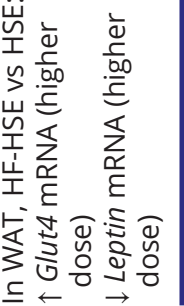

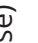

$\frac{\bar{c}}{\frac{10}{}}$ 
polypeptide and gut peptides (glucose-dependent insulinotropic polypeptides GIP, active amylin and active glucagon-like peptide GLP-1 and GLP-2) (Cani et al. 2009). Interesting, counteracting GLP-1 effect (by antagonists or in GLP-1 receptor knock-out mice) blunted the improvement of appetite and glucose homeostasis by OFS, supporting the importance of the modulation of the gut endocrine function to explain systemic effect of inulin-type fructans (ITF) prebiotics. In addition to their impact on Bifidobacterium spp. and adiposity, OFS also improved the intramuscular lipids accumulation in $\mathrm{ob} / \mathrm{ob}$ mice, attributed to a reduction of muscle triglycerides content (Everard et al. 2011). These beneficial impacts also occurred in obese rats in which an OFS-enriched inulin mixture improved adiposity, steatosis and lean mass, and increased GIP and GLP-1 levels (Kumar et al. 2016). In addition, short-chain fructo-oligosaccharides administration in humanized gnotobiotic mice fed a HF confirmed the expansion of Bifidobacterium spp. and the control of adiposity, this being associated with a reduction of plasma leptin and adiponectin (Respondek et al. 2013). However, the regulation of plasma triglycerides level by ITF is more controversial (Everard et al. 2011, Respondek et al. 2013, Kumar et al. 2016).

The mechanisms of action of ITF is often related to their fermentation into SCFA that lead researchers to analyze their influence on molecular targets of SCFA. For instance, one possible mechanism of ITF action could be the regulation of G protein-coupled receptor-3 (GPR43/ FFAR2) (Dewulf et al. 2011). The activation of GPR43 receptor, upon a HF diet, drives the inhibition of lipolysis and the stimulation of adipocytes differentiation. Interestingly, OFS reduced the expression of Gpr43 in subcutaneous adipose tissue (SAT) and reduced both the expression of cluster of differentiation $36(\mathrm{Cd} 36)$ and lipoprotein lipase ( $\mathrm{Lpl}$; involved in lipid accumulation in adipocytes), and the mRNA levels of $a P 2$ and CEBP- $\alpha$ (two markers of adipocytes differentiation) contributing to the anti-obesogenic effect of OFS (Dewulf et al. 2011). In addition to the effect reported in SAT, inulin also decreased $C d 36$ expression, in the jejunum of western diet fed mice (Hiel et al. 2018) and in the skeletal muscles of mice fed a western diet supplemented with gluten (Olivares et al. 2019), suggesting a role of ITF prebiotic in the regulation of FA uptake in several tissues. Another possible mechanism is an improvement of brown adipose tissue (BAT) activity and/or activation of 'browning' process in the WAT by ITF. Indeed, inulin increased the mRNA levels of Ppargc-1 $\alpha, U c p 1$ and Cidea, three markers of adipocyte 'browning' in SAT, and stimulated the activity of the cytochrome c oxidase in the BAT, suggesting a higher mitochondrial oxidative capacity in this tissue (Weitkunat et al. 2017). Interestingly, the basal gut microbiota influences the response to ITF in terms of microbial changes and metabolic effects in the peripheral tissues (Rodriguez et al. 2020). Indeed, the inulin effects on fat mass expansion, BAT thermogenesis and intramuscular lipids accumulation differently occur in groups of mice inoculated with the feces from different obese donors. In humans, fat oxidation was increased in the early postprandial phase in overweight to obese men consuming high-fat milkshake enriched in inulin, compared to a maltodextrin-based placebo (van der Beek et al. 2018). Finally, the impact of ITF on the adiposity and the growth of Bifidobacterium spp. has been confirmed in obese women and in a multicenter-clinical study performed in obese individuals after 3 months of supplementation (Dewulf et al. 2013), as well as upon a supplementation of FOS-enriched inulin during 16 weeks in overweight or obese children (Nicolucci et al. 2017).

In addition to the effects of ITF, several other food components, responding to the definition of dietary fibers and considered as prebiotic or prebiotic candidates, are of interest in the management of diet-induced obesity. It is the case of gluco-oligosacharides (GOS), $\beta$-galactooligosaccharides ( $\beta$-GAL) or mannan-oligosaccharides (MOS) (Serino et al. 2012, Wang et al. 2018b, Mistry et al. 2020). For instance, GOS, $\beta-G A L$ and MOS improved the expansion of white adipose tissue, insulin sensitivity or glucose tolerance, and plasma triglycerides in $\mathrm{HF}$ or western diet fed mice (Serino et al. 2012, Wang et al. $2018 b$, Mistry et al. 2020). $\beta$-GAL and MOS modulate the gut microbiota by favoring the growth of Bifidobacterium spp. and A. muciniphila (Wang et al. 2018b, Mistry et al. 2020). Finally, GOS and MOS could regulate the levels of some adipokines in plasma and WAT (Serino et al. 2012, Wang et al. 2018b). Furthermore, supplementation with milk oligosaccharides in HF-fed mice also affected the WAT since it decreased the number of crown-like structures in mesenteric adipose tissue, a marker of dead adipocytes and macrophages infiltration, which increases in obesity (Cinti et al. 2005, Hamilton et al. 2017).

Supplementation with chitin-glucan, another dietary fiber, increased the number of Bifidobacterium spp., Roseburia spp. and E. rectale/C. coccoides group in HF-fed mice, these effects on the gut microbiota were associated with a decreased adiposity and an improvement of glucose tolerance (Neyrinck et al. 2012a). The dietary fibers present in cereals are also of interest. Similar changes were found with arabinoxylan (AX) in HF-fed mice since 4 or 


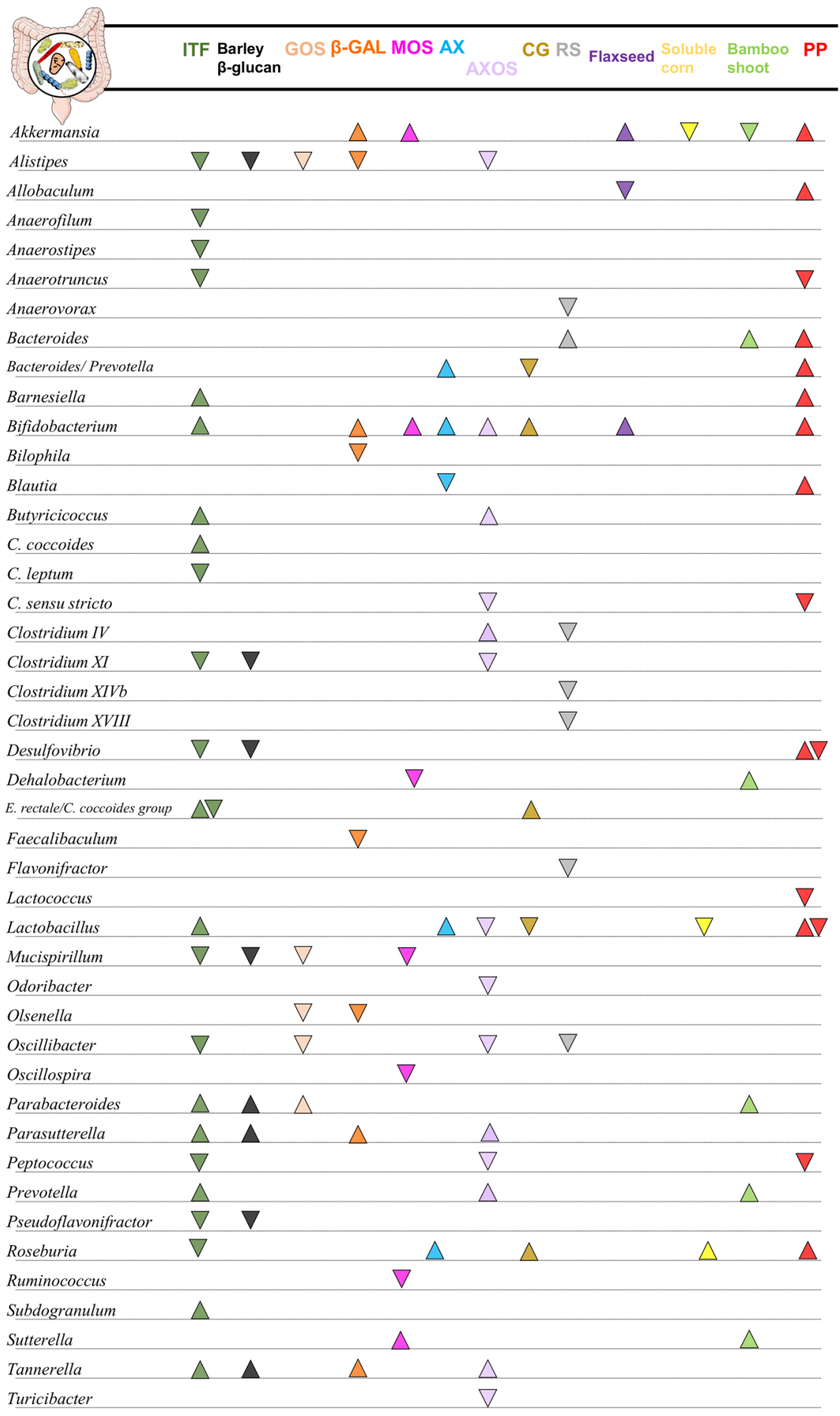

Genera decreased $(\boldsymbol{\nabla})$ or increased $(\boldsymbol{\Delta})$ by fibers/prebiotics

\section{Figure 1}

Effects of prebiotics on the gut microbiota composition in rodent models of obesity. The figure summarized the changes observed on the gut microbiota, at the genus level following a supplementation with prebiotics in obese mice. Genera increased $(\boldsymbol{\Lambda})$ or decreased $(\boldsymbol{\nabla})$ by prebiotics are listed. Each prebiotics is defined by a specific color, as indicated in the figure. ITF, inulin-type fructans; $\beta$-GAL, $\beta$-galactooligosaccharides; GOS, gluco-oligosachharides; MOS, mannan-oligosaccharides; AX, arabinoxylan; AXOS, arabinoxylan oligosaccharides; CG, chitin-glucan; RS, resistant starch; PP, polyphenols.
10 weeks of supplementation was sufficient to improve body weight and adiposity and stimulates the growth of Bifidobacterium (after 4 weeks) and Roseburia genera (independently of duration) (Neyrinck et al. 2011, Sarma et al. 2018). Interestingly, this cereal dietary fiber (DF) was able to down-regulate inflammation, lipogenesis, FA oxidation and adipocytes differentiation in adipose tissues after a short- or long-term administration. The hydrolysis of AX generates arabinoxylan oligosaccharides (AXOS) which also exhibit interesting prebiotic properties. Indeed, 


\begin{tabular}{|l|l|l|l|l|}
\hline $\begin{array}{l}\text { Journal of } \\
\text { Endocrinology }\end{array}$ & $\begin{array}{l}\text { J Rodriguez and } \\
\text { N M Delzenne }\end{array}$ & $\begin{array}{l}\text { Gut microbiome, fat mass and } \\
\text { skeletal muscles }\end{array}$ & $\mathbf{2 4 9 : 1}$ & R16 \\
\hline
\end{tabular}

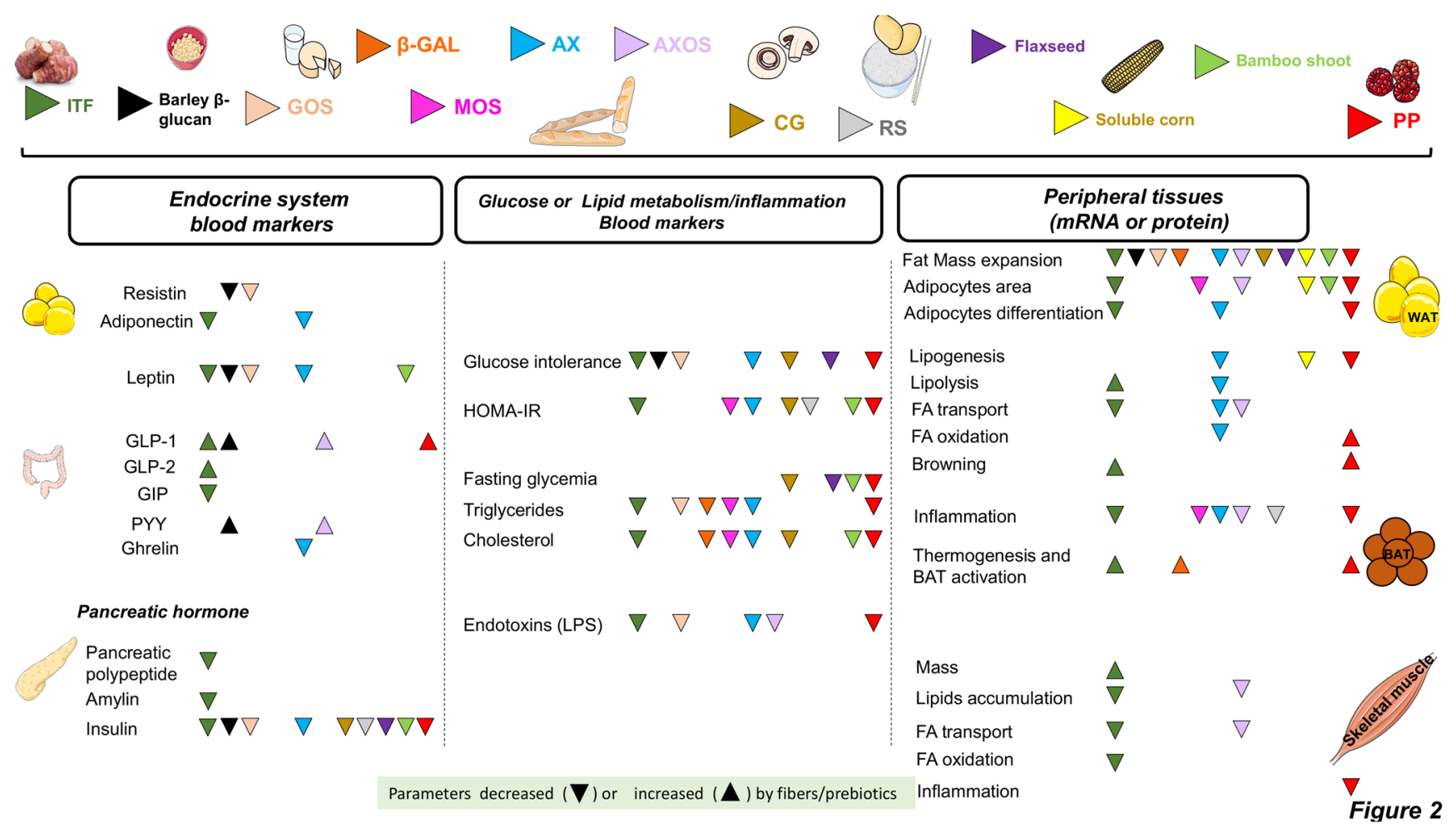

Figure 2

Effects of prebiotics on hormones, adipose tissue and skeletal muscle in rodent models of obesity. The figure summarized the effects of prebiotics on the parameters measured in serum, adipose tissues and skeletal muscle in obese mice. For each parameter, increased $(\boldsymbol{\Lambda})$ or decreased effects $(\boldsymbol{\nabla})$ of prebiotics are shown. Each prebiotics is defined by a specific color, as indicated in the figure. ITF, inulin-type fructans; $\beta$-GAL, $\beta$-galacto-oligosaccharides; GOS, gluco-oligosachharides; MOS, mannan-oligosaccharides; AX, arabinoxylan; AXOS, arabinoxylan oligosaccharides; CG, chitin-glucan; RS, resistant starch; PP, polyphenols.

8 weeks of AXOS supplementation in HF- and western (HF/HS) diet-fed mice led to a common signature that was an increase of Bifidobacterium spp. (Neyrinck et al. 2012b, Suriano et al. 2017, Olivares et al. 2019). In addition to their impact on the gut microbiota, AXOS can also regulated both body weight gain and fat accumulation (Neyrinck et al. 2012b, Olivares et al. 2019). In a HF model, AXOS increased the plasma levels of satiating hormones GLP-1 and (PYY) (Neyrinck et al. 2012b). Interestingly, in a western diet supplemented with gluten, which worsens the metabolic alterations, AXOS decreased inflammation in white adipose tissue and limited the intramuscular lipids accumulation (Olivares et al. 2019). Furthermore, the supplementation with AXOS allowed a significant reduction of $\mathrm{Cd} 36$ involved in FA transport in both adipose tissue and skeletal muscle (Olivares et al. 2019).

Barley $\beta$-glucan addition in a HF diet also improved the WAT expansion in mice, associated with an increased plasma levels of satiating hormones PYY and GLP-1, thus regulating food intake (Drew et al. 2018, Miyamoto et al. 2018). Moreover, addition of barley $\beta$-glucan in a HF diet reduced the level of plasma insulin (Drew et al. 2018, Miyamoto et al. 2018).

Supplementation with some other DF (bamboo shoot, soluble corn and flaxseed fibers) can also decrease the body weight gain, associated with an improvement of adipose tissues mass (Li et al. 2016, Wang et al. 2018a, Arora et al. 2019).

Finally, similarly to ITF supplementation, barley kernel-based bread (containing a ratio of 13\% non-starch polysaccharides $/ 11 \%$ resistant starch (Nilsson et al. 2015)) differentially modulate the gut microbiota and metabolic parameters in a cohort of volunteers (KovatchevaDatchary et al. 2015). Indeed, after three days of barley kernel-based bread consumption, the authors observed inter-individual variability for the improvement of glucose metabolism. Subjects exhibiting an improvement of glucose and insulin profiles are characterized by an enrichment of fecal Prevotella copri after barley kernelbread supplementation.

Regarding the impact of resistant starch (RS), one study previously reported that increased level of GLP-1 https://joe.bioscientifica.com

https://doi.org/10.1530/JOE-20-0499
(C) 2021 Society for Endocrinology Published by Bioscientifica Ltd. Printed in Great Britain 
and PYY by RS is important to mediate the effect of RS on body fat accumulation in mice (Zhou et al. 2015). Indeed, the anti-obesity effects of RS were not found in mice daily injected with a PYY receptor antagonist and in GLP-1-receptor deficient mice. However, this study did not address the impact of RS on the gut microbiota. In another study, Bindels et al. showed that RS feeding improved insulin sensitivity (especially the chemically modified RS type 4) in conventionalized mice, but this improvement also occurred in absence of gut microbiota - in germ-free mice-, supporting a microbiota-independent mechanism to explain the improvement of metabolic disorders by RS (Bindels et al. 2017). Although the macrophages markers were reduced by RS type 2 (granular starches) and type 4 in SAT and VAT (independently of the presence of gut microbiota), the authors did not report any effects of RS on adiposity and plasma GLP-1 or PYY.

Finally, in humans, 84 days of supplementation with different formula enriched in diverse structures of fibers ( $\beta$-glucan, arabinoxylan, cellulose, resistant starch, gums, oligosaccharides, inulin and resistant dextrin) improved glucose homeostasis by reducing both the fasting blood glucose and glycated hemoglobin HBA1c in type 2 diabetes (T2D) patients, and promoted the growth of SCFA-producing strains (Zhao et al. 2018). In this study, the trend of increased fecal acetate and butyrate concentrations in the treated group coincided with a significantly greater postprandial GLP-1 area under the curve, and a higher level of fasting PYY.

Some polyphenols also respond to the definition of prebiotics (Gibson et al. 2017). Some studies demonstrated the impact of these phenolic compounds on the gut microbiota and their metabolic consequences. Using the same strategy, we summarize recent discovery about the impact of polyphenols on both gut microbiota composition and adipose tissue or skeletal muscle (Table 1 ). The majority of studies, related to the impact of polyphenols on gut microbiota and metabolic alterations induced by a HF diet, demonstrated beneficial effects of polyphenol on adipose tissues (Qiao et al. 2014, Anhe et al. 2015, Collins et al. 2016, Gao et al. 2018, Liao et al. 2018, Anhe et al. 2019, Jiao et al. 2019, Sheng et al. 2019, DiezEchave et al. 2020, Wang et al. 2020). However, little is known about the regulations observed in skeletal muscles in these conditions. Regarding the adipose tissues, it seems evident that polyphenols supplementation during a HF diet impact the homeostasis of this organ. Most of the in vivo studies reported an increase of markers (such as Ucp1, Ppargc1a, Ppary, Sirt1, Prdm16 or Cidea) involved in the browning of WAT after polyphenols supplementation during a HF diet (Gao et al. 2018, Liao et al. 2018, Anhe et al. 2019, Zhou et al. 2019, Wang et al. 2020). Some studies also demonstrated the induction by polyphenols of specific markers of BAT activation, including energy expenditure or thermogenesis (Ucp1, Ppary, Cpt $\alpha$, Ppargc1 $\alpha$ and $\beta, C E B P / \alpha$ and $\beta$ ) (Anhe et al. 2019, Sheng et al. 2019). Finally, polyphenols also modulate the lipogenesis or FA oxidation, leading to a decreased mRNA expression of Cpt1, Ppary and Fasn in WAT (Qiao et al. 2014, Collins et al. 2016, Jiao et al. 2019, Wang et al. 2020). In addition, they exert antiinflammatory properties in WAT and in skeletal muscles of HF-fed mice (Dao et al. 2011, Neyrinck et al. 2013, Collins et al. 2016, Gao et al. 2018). Interestingly, since a great improvement in both glucose and lipid metabolism by polyphenols are observed in these different studies, those effects are not necessarily linked to a change in adiposity (Neyrinck et al. 2013, Van Hul et al. 2018, Jiao et al. 2019). The signature of polyphenols on the gut microbiota appears complex due to the large diversity of this class of compounds. However, a regulation of some bacteria associated with beneficial outcomes (and known to be increased with other kind of prebiotics) was also observed in some polyphenols studies, such as Bifidobacterium spp., Roseburia spp. or Akkermansia (Neyrinck et al. 2013, Qiao et al. 2014, Anhe et al. 2015, Gao et al. 2018, Van Hul et al. 2018, Anhe et al. 2019, Jiao et al. 2019, Sheng et al. 2019).

\section{Bacterial metabolites linking prebiotic intake and metabolic feature in gut hormones, fat and muscle tissues}

Prebiotics alter the composition of the gut microbiota, and thereby, or through their fermentation, can modulate the profile of the microbial metabolites prone to exert metabolic effects in the gut, or at distance in peripheral organs. Microbial SCFA production is essential for gut integrity but also for the modulation of host metabolic health through a range of tissue-specific mechanisms related to appetite regulation, energy expenditure, glucose homeostasis and immunomodulation (Blaak et al. 2020). Some studies support a beneficial role for some SCFA in the adipose tissue, the skeletal muscle, as well as in the liver, that can contribute to improved insulin sensitivity (for review Canfora et al. 2015, Blaak et al. 2020).

However, all SCFA exhibit different effects and while butyrate, acetate seem to be associated with beneficial impact on metabolism, the microbialderived succinate could contribute to the progression of 
insulin resistance. Propionate and butyrate can activate intestinal gluconeogenesis and released glucose initiates a neural signal leading to satiety and metabolic benefits including insulin sensitivity (Blaak et al. 2020). SCFA can be also important regulators of pancreatic insulin secretion and $\beta$-cell functioning, such as demonstrated with propionate (Blaak et al. 2020).

As already mentioned, SCFA are mainly produced from the carbohydrate and DF fermentation and are ligands of G-protein coupled receptors (GPR41 and GPR43). By binding these receptors, SCFA can regulate glucose homeostasis through the control of satiety hormones production (PYY, GLP-1) and adiposity through peroxisome proliferator-activated receptor $\gamma$ (PPAR $\gamma$ )dependent mechanisms (Dewulf et al. 2011, Canfora et al. 2015).

Six months of acetate injections in obese rats improved the expression of myoglobin, glucose transporter 4 (Glut4) and activated AMPK protein by increasing the AMP/ATP ratio in abdominal muscles (Yamashita et al. 2009). In addition, acetate induced smaller lipids droplets in both SAT and BAT from obese rats. Surprisingly, SCFA-mediated activation of GPR43 seemed to suppress insulin signaling only in adipocytes, leading to an inhibition of fat accumulation, but not in the liver and skeletal muscles, (Kimura et al. 2013). Butyrate also had a beneficial impact on insulin sensitivity and adiposity in HF-fed mice, which coincided with an increased FA oxidation in skeletal muscles and increased thermogenesis in BAT (Gao et al. 2009). Acetate and propionate also prevented diet-induced metabolic alterations (body weight gain, adiposity, hepatic steatosis, insulin resistance) in mice, acetate being the sole to enhance markers of 'beiging' in WAT and increased body temperature reflecting oxidation processes (Weitkunat et al. 2017). A recent study also highlighted that SCFA mixture can control both muscle mass and strength (Lahiri et al. 2019). Indeed, compared to untreated GF mice, the addition of SCFA mixture in the drinking water of treated GF mice increased gastrocnemius weight, hindlimb grip strength and the mRNA expression of $M y o D$ in tibialis, a marker involved in myogenesis (Lahiri et al. 2019).

In addition to the SCFA, the chronic administration of 4-cresol, a metabolite produced from amino-acid fermentation, in HF-fed mice reduced body weight gain, adiposity, glucose intolerance and liver fat content (Brial et al. 2020). This was associated with an improvement of insulin secretion and a stimulation of pancreatic $\beta$-cell function. Other secondary metabolites can be produced by the gut microbiota from polyphenols and regulates metabolic processes in adipose tissues or skeletal muscles. Indeed, urolithin A produced by the gut microbiota from ellagic acid, can prevent HF-induced and genetic obesity in mice, as well as dysfunctional glucose homeostasis and this was associated with enhanced BAT thermogenesis and white fat browning (Xia et al. 2020). Moreover, independently of metabolic alterations, urolithin A can promote the skeletal muscle mitochondrial function in preclinical models of aging and elderly individuals (Ryu et al. 2016, Andreux et al. 2019). In addition, the isoform urolithin B can also impact skeletal muscle health by regulating insulin signaling and promote protein synthesis (Rodriguez et al. 2017).

Bile acids (BA) are endogenous steroid molecules derived from cholesterol, produced in hepatocytes and then converted through deconjugation and reduction, into secondary BA by gut microbiota. BA can regulate host metabolism through Takeda G-protein receptor 5 (TGR5) and Farnesoid X receptor (FXR), both expressed in various tissues (for review Chavez-Talavera et al. 2017, Molinaro et al. 2018). Indeed, activation of FXR by BA decreases hepatic lipogenesis and hepatic inflammation, increases adipocytes differentiation and function in WAT, and enhances insulin secretion by pancreas. On the other hand, TGR5 stimulation enhances the intestinal production and secretion of GLP1, the insulin secretion by pancreas, and increases the energy expenditure and activation of BAT. Both receptor are able to modulate the intestinal GLP-1 secretion, but in an opposite way.

Of note, not all bacterial metabolites derived from foods are associated with beneficial effects on metabolism. For instance, imidazole propionate produced from histidine can directly impair glucose tolerance and insulin signaling (Koh et al. 2018). This metabolite is increased during type 2 diabetes and inhibits insulin receptor substrate 1 and/or 2 in tissues with a high metabolic activity such as liver, WAT or soleus muscle. Furthermore, the bacterial metabolite trimethylamine (produced by gut microbiota mainly from red meat) can be metabolized by the host hepatic enzyme flavin-containing monooxygenase 3 (FMO3) to produce trimethylamine-N-oxide (TMAO), a product exhibiting deleterious effects on host metabolism such as atherosclerosis (Koeth et al. 2013). Interestingly, genetic deletion of FMO3 enzyme conferred protection against obesity in mice, through the stimulation of WAT beiging, meaning that decreased TMAO induced a metabolically active beige adipose tissue (Schugar et al. 2017).

Taken together, these data strongly support the hypothesis that some gut-related metabolites from the diet could link the gut microbiota with molecular events 
occurring in circulation and peripheral tissues for the maintenance of energy homeostasis.

\section{Conclusion and future prospects}

In the present review, we have discussed about the importance to modulate the gut microbiota composition in order to control the energy homeostasis in adipose tissues vs skeletal muscle. It is surprising to note that few studies have investigated the response of skeletal muscles after dietary intervention using prebiotics. However, an important factor to take into consideration beside the nutrition is, obviously, the physical activity status. An elegant recent study highlighted that the gut microbiota fermentation determines the efficacy of exercise in the control of glucose metabolism in prediabetic individuals (Liu et al. 2020). By demonstrating the variability of glycemic response to exercise, the authors showed that the high-responders for amelioration of insulin resistance were characterized by a higher carbohydrate fermentation (increased SCFA production) and amino acid catabolism (decreased BCAA availability). Interestingly, fecal microbial transplantation from responders in obese mice reproduced the beneficial effects of exercise on insulin resistance.

Whether the fermentation ability of the gut microbiota can influence the metabolic response to exercise, thus we hypothesize that the diet can also modulate the efficacy of exercise. Indeed, promoting fermentable foods can be a good strategy to increase the substrate for fermentation and improve the beneficial effects of exercise on energy metabolism.

Finally, the therapeutic perspectives issued from the better knowledge of gut -muscle-adipose tissue dialogue are not restricted to the problem of overweight and related diseases. Due to their beneficial impact on several host molecular process, the use of prebiotic cannot be limited to the metabolic alterations, but also envisioned in different (physio-)pathological situations such as cancer cachexia (for review Potgens et al. 2018). Indeed, pectin derived oligosaccharides (POS) and inulin have been evaluated in leukemic mouse model of cachexia (Bindels et al. 2015). Very nicely, whereas inulin was more efficient to decrease hepatic cancer cell invasion, POS supplementation improves metabolic phenotype (to a higher extent than inulin), and reduced fat mass loss. On a mechanistic point, POS reversed the induction by cachexia of both Cpt1a and Ppargc1 $\alpha$ mRNA in SAT, two markers controlling FA oxidation.
Interestingly, during cachexia, POS enhanced (better than inulin) the growth of Bifidobacterium spp., Roseburia spp. and Bacteroides/Prevotella spp. In addition, combining both prebiotic with probiotic could also be a promising therapy in this context. Indeed, a synbiotic containing inulin-type fructans and live Lactobacillus reuteri administered to leukemic mice reduced cachexia, and more specifically the loss of skeletal muscle mass (Bindels et al. 2016). This was linked to a reduction of both cathepsin L and LC3 mRNA in muscles, two markers involved in the protein degradation in skeletal muscles. These studies based on prebiotics and performed in another context reinforces the hypothesis that metabolic regulations occur between different organs rely from the gut microbiota modifications.

To conclude, future studies should be addressed in order to investigate, among other approaches, the interest of the different tools able to modulate the gut microbiota (FMT, prebiotics, probiotics, synbiotics) to face problems of malnutrition (covering both overeating and undernutrition) worldwide. The fact that prebiotics and dietary fibers may improve concomitantly the endocrine function, and energy metabolism in most organs (liver, muscle adipose tissues....), support their interest as drivers of systems biology in the context of a healthy diet.

\section{Declaration of interest}

The authors declare that there is no conflict of interest that could be perceived as prejudicing the impartiality of this review.

\section{Funding}

N M D is a recipient of grants from the Service Public de Wallonie (SPW-EER, convention 1610365, FiberTAG project from European Joint Programming Initiative 'A Healthy Diet for a Healthy Life'), from the Fonds de la Recherche Scientifique (FRS-FNRS, convention PINT-MULTI R.8013.19 (NEURON, call 2019) and convention PDR T.0068.19) and from the Fédération WallonieBruxelles (Action de Recherche Concertée ARC18-23/092).

\section{References}

Alard J, Lehrter V, Rhimi M, Mangin I, Peucelle V, Abraham AL, Mariadassou M, Maguin E, Waligora-Dupriet AJ, Pot B, et al. 2016 Beneficial metabolic effects of selected probiotics on dietinduced obesity and insulin resistance in mice are associated with improvement of dysbiotic gut microbiota. Environmental Microbiology 18 1484-1497. (https://doi.org/10.1111/1462-2920.13181)

Allin KH, Tremaroli V, Caesar R, Jensen BAH, Damgaard MTF, Bahl MI, Licht TR, Hansen TH, Nielsen T, Dantoft TM, et al. 2018 Aberrant intestinal microbiota in individuals with prediabetes. Diabetologia 61 810-820. (https://doi.org/10.1007/s00125-018-4550-1)

Andreux PA, Blanco-Bose W, Ryu D, Burdet F, Ibberson M, Aebischer P, Auwerx J, Singh A \& Rinsch C 2019 The mitophagy activator 
urolithin A is safe and induces a molecular signature of improved mitochondrial and cellular health in humans. Nature Metabolism 1 595-603. (https://doi.org/10.1038/s42255-019-0073-4)

Anhe FF, Roy D, Pilon G, Dudonne S, Matamoros S, Varin TV, Garofalo C, Moine Q, Desjardins Y, Levy E, et al. 2015 A polyphenol-rich cranberry extract protects from diet-induced obesity, insulin resistance and intestinal inflammation in association with increased Akkermansia spp. population in the gut microbiota of mice. Gut $\mathbf{6 4}$ 872-883. (https://doi.org/10.1136/gutjnl-2014-307142)

Anhe FF, Nachbar RT, Varin TV, Trottier J, Dudonne S, Le Barz M, Feutry P, Pilon G, Barbier O, Desjardins Y, et al. 2019 Treatment with camu camu (Myrciaria dubia) prevents obesity by altering the gut microbiota and increasing energy expenditure in diet-induced obese mice. Gut 68 453-464. (https://doi.org/10.1136/gutjnl-2017-315565)

Arora T, Rudenko O, Egerod KL, Husted AS, Kovatcheva-Datchary P, Akrami R, Kristensen M, Schwartz TW \& Backhed F 2019 Microbial fermentation of flaxseed fibers modulates the transcriptome of GPR41-expressing enteroendocrine cells and protects mice against diet-induced obesity. American Journal of Physiology: Endocrinology and Metabolism 316 E453-E463. (https://doi.org/10.1152/ ajpendo.00391.2018)

Backhed F, Ding H, Wang T, Hooper LV, Koh GY, Nagy A, Semenkovich CF \& Gordon JI 2004 The gut microbiota as an environmental factor that regulates fat storage. PNAS 101 15718-15723. (https://doi. org/10.1073/pnas.0407076101)

Backhed F, Manchester JK, Semenkovich CF \& Gordon JI 2007 Mechanisms underlying the resistance to diet-induced obesity in germ-free mice. PNAS 104 979-984. (https://doi.org/10.1073/ pnas.0605374104)

Bagarolli RA, Tobar N, Oliveira AG, Araujo TG, Carvalho BM, Rocha GZ, Vecina JF, Calisto K, Guadagnini D, Prada PO, et al. 2017 Probiotics modulate gut microbiota and improve insulin sensitivity in DIO mice. Journal of Nutritional Biochemistry 50 16-25. (https://doi.org/10.1016/j. jnutbio.2017.08.006)

Bindels LB, Neyrinck AM, Salazar N, Taminiau B, Druart C, Muccioli GG, Francois E, Blecker C, Richel A, Daube G, et al. 2015 Non digestible oligosaccharides modulate the gut microbiota to control the development of leukemia and associated cachexia in mice. PLoS ONE 10 e0131009. (https://doi.org/10.1371/journal.pone.0131009)

Bindels LB, Neyrinck AM, Claus SP, Le Roy CI, Grangette C, Pot B, Martinez I, Walter J, Cani PD \& Delzenne NM 2016 Synbiotic approach restores intestinal homeostasis and prolongs survival in leukaemic mice with cachexia. ISME Journal 10 1456-1470. (https:// doi.org/10.1038/ismej.2015.209)

Bindels LB, Segura Munoz RR, Gomes-Neto JC, Mutemberezi V, Martinez I, Salazar N, Cody EA, Quintero-Villegas MI, Kittana H, de Los Reyes-Gavilan CG, et al. 2017 Resistant starch can improve insulin sensitivity independently of the gut microbiota. Microbiome $\mathbf{5}$ 12. (https://doi.org/10.1186/s40168-017-0230-5)

Blaak EE, Canfora EE, Theis S, Frost G, Groen AK, Mithieux G, Nauta A Scott K, Stahl B, van Harsselaar J, et al. 2020 Short chain fatty acids in human gut and metabolic health. Beneficial Microbes 11 411-455. (https://doi.org/10.3920/BM2020.0057)

Brial F, Alzaid F, Sonomura K, Kamatani Y, Meneyrol K, Le Lay A, Pean N, Hedjazi L, Sato TA, Venteclef N, et al. 2020 The natural metabolite 4-cresol improves glucose homeostasis and enhances beta-cell function. Cell Reports 30 2306.e5-2320.e5. (https://doi.org/10.1016/j. celrep.2020.01.066)

Caesar R, Reigstad CS, Backhed HK, Reinhardt C, Ketonen M, Lunden GÖ, Cani PD \& Backhed F 2012 Gut-derived lipopolysaccharide augments adipose macrophage accumulation but is not essential for impaired glucose or insulin tolerance in mice. Gut 61 1701-1707. (https://doi. org/10.1136/gutjnl-2011-301689)

Caesar R, Tremaroli V, Kovatcheva-Datchary P, Cani PD \& Backhed F 2015 Crosstalk between gut microbiota and dietary lipids aggravates WAT inflammation through TLR signaling. Cell Metabolism 22 658-668. (https://doi.org/10.1016/j.cmet.2015.07.026)

Canfora EE, Jocken JW \& Blaak EE 2015 Short-chain fatty acids in control of body weight and insulin sensitivity. Nature Reviews: Endocrinology 11 577-591. (https://doi.org/10.1038/nrendo.2015.128)

Canfora EE, Meex RCR, Venema K \& Blaak EE 2019 Gut microbial metabolites in obesity, NAFLD and T2DM. Nature Reviews: Endocrinology 15 261-273. (https://doi.org/10.1038/s41574-019-0156-z)

Cani PD, Amar J, Iglesias MA, Poggi M, Knauf C, Bastelica D, Neyrinck AM, Fava F, Tuohy KM, Chabo C, et al. 2007a Metabolic endotoxemia initiates obesity and insulin resistance. Diabetes 56 1761-1772. (https://doi.org/10.2337/db06-1491)

Cani PD, Neyrinck AM, Fava F, Knauf C, Burcelin RG, Tuohy KM, Gibson GR \& Delzenne NM 2007b Selective increases of bifidobacteria in gut microflora improve high-fat-diet-induced diabetes in mice through a mechanism associated with endotoxaemia. Diabetologia 50 2374-2383. (https://doi.org/10.1007/s00125-007-0791-0)

Cani PD, Possemiers S, Van de Wiele T, Guiot Y, Everard A, Rottier O, Geurts L, Naslain D, Neyrinck A, Lambert DM, et al. 2009 Changes in gut microbiota control inflammation in obese mice through a mechanism involving GLP-2-driven improvement of gut permeability. Gut 58 1091-1103. (https://doi.org/10.1136/gut.2008.165886)

Cerdo T, Garcia-Santos JA, G Bermúdez M \& Campoy C 2019 The role of probiotics and prebiotics in the prevention and treatment of obesity. Nutrients 11 635. (https://doi.org/10.3390/nu11030635)

Chavez-Talavera O, Tailleux A, Lefebvre P \& Staels B 2017 Bile acid control of metabolism and inflammation in obesity, Type 2 diabetes, dyslipidemia, and nonalcoholic fatty liver disease. Gastroenterology 152 1679.e3-1694.e3. (https://doi.org/10.1053/j.gastro.2017.01.055)

Cinti S, Mitchell G, Barbatelli G, Murano I, Ceresi E, Faloia E, Wang S, Fortier M, Greenberg AS \& Obin MS 2005 Adipocyte death defines macrophage localization and function in adipose tissue of obese mice and humans. Journal of Lipid Research 46 2347-2355. (https://doi. org/10.1194/jlr.M500294-JLR200)

Collins B, Hoffman J, Martinez K, Grace M, Lila MA, Cockrell C, Nadimpalli A, Chang E, Chuang CC, Zhong W, et al. 2016 A polyphenol-rich fraction obtained from table grapes decreases adiposity, insulin resistance and markers of inflammation and impacts gut microbiota in high-fat-fed mice. Journal of Nutritional Biochemistry 31 150-165. (https://doi.org/10.1016/j.jnutbio.2015.12.021)

Cotillard A, Kennedy SP, Kong LC, Prifti E, Pons N, Le Chatelier E, Almeida M, Quinquis B, Levenez F, Galleron N, et al. 2013 Dietary intervention impact on gut microbial gene richness. Nature $\mathbf{5 0 0}$ 585-588. (https://doi.org/10.1038/nature12480)

da Silva TF, Casarotti SN, de Oliveira GLV \& Penna ALB 2020 The impact of probiotics, prebiotics, and synbiotics on the biochemical, clinical, and immunological markers, as well as on the gut microbiota of obese hosts. Critical Reviews in Food Science and Nutrition 61 337-355. (https://doi.org/10.1080/10408398.2020.1733483)

Dao TM, Waget A, Klopp P, Serino M, Vachoux C, Pechere L, Drucker DJ, Champion S, Barthelemy S, Barra Y, et al. 2011 Resveratrol increases glucose induced GLP-1 secretion in mice: a mechanism which contributes to the glycemic control. PLOS ONE 6 e20700. (https://doi. org/10.1371/journal.pone.0020700)

Depommier C, Everard A, Druart C, Plovier H, Van Hul M, VieiraSilva S, Falony G, Raes J, Maiter D, Delzenne NM, et al. 2019 Supplementation with Akkermansia muciniphila in overweight and obese human volunteers: a proof-of-concept exploratory study. Nature Medicine 25 1096-1103. (https://doi.org/10.1038/s41591-0190495-2)

Depommier C, Van Hul M, Everard A, Delzenne NM, De Vos WM \& Cani PD 2020 Pasteurized Akkermansia muciniphila increases wholebody energy expenditure and fecal energy excretion in diet-induced obese mice. Gut Microbes 11 1231-1245. (https://doi.org/10.1080/194 90976.2020.1737307) https://joe.bioscientifica.com

https://doi.org/10.1530/JOE-20-0499 (c) 2021 Society for Endocrinology Published by Bioscientifica Ltd. Printed in Great Britain 
Dewulf EM, Cani PD, Neyrinck AM, Possemiers S, Van Holle A, Muccioli GG, Deldicque L, Bindels LB, Pachikian BD, Sohet FM, et al. 2011 Inulin-type fructans with prebiotic properties counteract GPR43 overexpression and PPARgamma-related adipogenesis in the white adipose tissue of high-fat diet-fed mice. Journal of Nutritional Biochemistry 22 712-722. (https://doi.org/10.1016/j. jnutbio.2010.05.009)

Dewulf EM, Cani PD, Claus SP, Fuentes S, Puylaert PG, Neyrinck AM, Bindels LB, de Vos WM, Gibson GR, Thissen JP, et al. 2013 Insight into the prebiotic concept: lessons from an exploratory, double blind intervention study with inulin-type fructans in obese women. Gut 62 1112-1121. (https://doi.org/10.1136/gutjnl-2012-303304)

Diez-Echave P, Vezza T, Rodriguez-Nogales A, Ruiz-Malagon AJ, HidalgoGarcia L, Garrido-Mesa J, Molina-Tijeras JA, Romero M, Robles-Vera I, Pimentel-Moral S, et al. 2020 The prebiotic properties of Hibiscus sabdariffa extract contribute to the beneficial effects in diet-induced obesity in mice. Food Research International 127 108722. (https://doi. org/10.1016/j.foodres.2019.108722)

Drew JE, Reichardt N, Williams LM, Mayer CD, Walker AW, Farquharson AJ, Kastora S, Farquharson F, Milligan G, Morrison DJ, et al. 2018 Dietary fibers inhibit obesity in mice, but host responses in the cecum and liver appear unrelated to fiber-specific changes in cecal bacterial taxonomic composition. Scientific Reports 8 15566. (https:// doi.org/10.1038/s41598-018-34081-8)

Everard A, Lazarevic V, Derrien M, Girard M, Muccioli GG, Neyrinck AM, Possemiers S, Van Holle A, Francois P, de Vos WM, et al. 2011 Responses of gut microbiota and glucose and lipid metabolism to prebiotics in genetic obese and diet-induced leptin-resistant mice. Diabetes 60 2775-2786. (https://doi.org/10.2337/db11-0227)

Everard A, Belzer C, Geurts L, Ouwerkerk JP, Druart C, Bindels LB, Guiot Y, Derrien M, Muccioli GG, Delzenne NM, et al. 2013 Cross-talk between Akkermansia muciniphila and intestinal epithelium controls diet-induced obesity. PNAS 110 9066-9071. (https://doi.org/10.1073/ pnas.1219451110)

Fan Y \& Pedersen O 2020 Gut microbiota in human metabolic health and disease. Nature Reviews in Microbiology 19 55-71. (https://doi. org/10.1038/s41579-020-0433-9)

Forslund K, Hildebrand F, Nielsen T, Falony G, Le Chatelier E, Sunagawa S, Prifti E, Vieira-Silva S, Gudmundsdottir V, Pedersen HK, et al. 2015 Disentangling type 2 diabetes and metformin treatment signatures in the human gut microbiota. Nature 528 262-266. (https://doi.org/10.1038/nature15766)

Gao Z, Yin J, Zhang J, Ward RE, Martin RJ, Lefevre M, Cefalu WT \& Ye J 2009 Butyrate improves insulin sensitivity and increases energy expenditure in mice. Diabetes 58 1509-1517. (https://doi. org/10.2337/db08-1637)

Gao X, Xie Q, Kong P, Liu L, Sun S, Xiong B, Huang B, Yan L, Sheng J \& Xiang H 2018 Polyphenol- and caffeine-rich postfermented Pu-erh tea improves diet-induced metabolic syndrome by remodeling intestinal homeostasis in mice. Infection and Immunity 86 e00601-17. (https:// doi.org/10.1128/IAI.00601-17)

Gibson GR, Hutkins R, Sanders ME, Prescott SL, Reimer RA, Salminen SJ, Scott K, Stanton C, Swanson KS, Cani PD, et al. 2017 Expert consensus document: the International Scientific Association for Probiotics and Prebiotics (ISAPP) consensus statement on the definition and scope of prebiotics. Nature Reviews: Gastroenterology and Hepatology 14 491-502. (https://doi.org/10.1038/nrgastro.2017.75)

Green M, Arora K \& Prakash S 2020 Microbial medicine: prebiotic and probiotic functional foods to target obesity and metabolic syndrome. International Journal of Molecular Sciences 21 2890. (https://doi. org/10.3390/ijms21082890)

Hamilton MK, Ronveaux CC, Rust BM, Newman JW, Hawley M, Barile D, Mills DA \& Raybould HE 2017 Prebiotic milk oligosaccharides prevent development of obese phenotype, impairment of gut permeability, and microbial dysbiosis in high fat-fed mice. American Journal of
Physiology: Gastrointestinal and Liver Physiology 312 G474-G487. (https://doi.org/10.1152/ajpgi.00427.2016)

Hiel S, Neyrinck AM, Rodriguez J, Pachikian BD, Bouzin C, Thissen JP, Cani PD, Bindels LB \& Delzenne NM 2018 Inulin improves postprandial hypertriglyceridemia by modulating gene expression in the small intestine. Nutrients 10 532. (https://doi.org/10.3390/ nu10050532)

Hill C, Guarner F, Reid G, Gibson GR, Merenstein DJ, Pot B, Morelli L, Canani RB, Flint HJ, Salminen S, et al. 2014 Expert consensus document. The International Scientific Association for Probiotics and Prebiotics Consensus Statement on the scope and appropriate use of the term probiotic. Nature Reviews: Gastroenterology and Hepatology 11 506-514. (https://doi.org/10.1038/nrgastro.2014.66)

Jiao X, Wang Y, Lin Y, Lang Y, Li E, Zhang X, Zhang Q, Feng Y, Meng X \& Li B 2019 Blueberry polyphenols extract as a potential prebiotic with anti-obesity effects on C57BL/6 J mice by modulating the gut microbiota. Journal of Nutritional Biochemistry 64 88-100. (https://doi. org/10.1016/j.jnutbio.2018.07.008)

Karlsson FH, Tremaroli V, Nookaew I, Bergstrom G, Behre CJ, Fagerberg B, Nielsen J \& Backhed F 2013 Gut metagenome in European women with normal, impaired and diabetic glucose control. Nature $\mathbf{4 9 8}$ 99-103. (https://doi.org/10.1038/nature12198)

Kayser BD, Prifti E, Lhomme M, Belda E, Dao MC, Aron-Wisnewsky J, MICRO-Obes Consortium, Kontush A, Zucker JD, Rizkalla SW, et al. 2019 Elevated serum ceramides are linked with obesity-associated gut dysbiosis and impaired glucose metabolism. Metabolomics 15140. (https://doi.org/10.1007/s11306-019-1596-0)

Kimura I, Ozawa K, Inoue D, Imamura T, Kimura K, Maeda T, Terasawa K, Kashihara D, Hirano K, Tani T, et al. 2013 The gut microbiota suppresses insulin-mediated fat accumulation via the short-chain fatty acid receptor GPR43. Nature Communications 4 1829. (https:// doi.org/10.1038/ncomms2852)

Koeth RA, Wang Z, Levison BS, Buffa JA, Org E, Sheehy BT, Britt EB, $\mathrm{Fu} \mathrm{X}, \mathrm{Wu}$ Y, Li L, et al. 2013 Intestinal microbiota metabolism of L-carnitine, a nutrient in red meat, promotes atherosclerosis. Nature Medicine 19 576-585. (https://doi.org/10.1038/nm.3145)

Koh A, Molinaro A, Stahlman M, Khan MT, Schmidt C, MannerasHolm L, Wu H, Carreras A, Jeong H, Olofsson LE, et al. 2018 Microbially produced imidazole propionate impairs insulin signaling through mTORC1. Cell 175 947.e17-961.e17. (https://doi. org/10.1016/j.cell.2018.09.055)

Kootte RS, Levin E, Salojarvi J, Smits LP, Hartstra AV, Udayappan SD, Hermes G, Bouter KE, Koopen AM, Holst JJ, et al. 2017 Improvement of insulin sensitivity after lean donor feces in metabolic syndrome is driven by baseline intestinal microbiota composition. Cell Metabolism 26 611.e6-619.e6. (https://doi.org/10.1016/j. cmet.2017.09.008)

Kovatcheva-Datchary P, Nilsson A, Akrami R, Lee YS, De Vadder F, Arora T, Hallen A, Martens E, Bjorck I \& Backhed F 2015 Dietary fiber-induced improvement in glucose metabolism is associated with increased abundance of Prevotella. Cell Metabolism 22 971-982. (https://doi.org/10.1016/j.cmet.2015.10.001)

Kumar SA, Ward LC \& Brown L 2016 Inulin oligofructose attenuates metabolic syndrome in high-carbohydrate, high-fat diet-fed rats. British Journal of Nutrition 116 1502-1511. (https://doi.org/10.1017/ S0007114516003627)

Lahiri S, Kim H, Garcia-Perez I, Reza MM, Martin KA, Kundu P, Cox LM, Selkrig J, Posma JM, Zhang H, et al. 2019 The gut microbiota influences skeletal muscle mass and function in mice. Science Translational Medicine 11 eaan5662. (https://doi.org/10.1126/ scitranslmed.aan5662)

Le Chatelier E, Nielsen T, Qin J, Prifti E, Hildebrand F, Falony G, Almeida M, Arumugam M, Batto JM, Kennedy S, et al. 2013 Richness of human gut microbiome correlates with metabolic markers. Nature 500 541-546. (https://doi.org/10.1038/nature12506) https://joe.bioscientifica.com

https://doi.org/10.1530/JOE-20-0499 (c) 2021 Society for Endocrinology Published by Bioscientifica Ltd. Printed in Great Britain 
Li X, Guo J, Ji K \& Zhang P 2016 Bamboo shoot fiber prevents obesity in mice by modulating the gut microbiota. Scientific Reports 632953. (https://doi.org/10.1038/srep32953)

Liao W, Yin X, Li Q, Zhang H, Liu Z, Zheng X, Zheng L \& Feng X 2018 Resveratrol-induced white adipose tissue browning in obese mice by remodeling fecal microbiota. Molecules 23 3356. (https://doi. org/10.3390/molecules23123356)

Liu Y, Wang Y, Ni Y, Cheung CKY, Lam KSL, Wang Y, Xia Z, Ye D, Guo J, Tse MA, et al. 2020 Gut microbiome fermentation determines the efficacy of exercise for diabetes prevention. Cell Metabolism 3177. e5-91.e5. (https://doi.org/10.1016/j.cmet.2019.11.001)

Mistry RH, Liu F, Borewicz K, Lohuis MAM, Smidt H, Verkade HJ \& Tietge UJF 2020 Long-term beta-galacto-oligosaccharides supplementation decreases the development of obesity and insulin resistance in mice fed a western-type diet. Molecular Nutrition and Food Research 64 e1900922. (https://doi.org/10.1002/ mnfr.201900922)

Miyamoto J, Watanabe K, Taira S, Kasubuchi M, Li X, Irie J, Itoh H \& Kimura I 2018 Barley beta-glucan improves metabolic condition via short-chain fatty acids produced by gut microbial fermentation in high fat diet fed mice. PLOS ONE 13 e0196579. (https://doi. org/10.1371/journal.pone.0196579)

Molinaro A, Wahlstrom A \& Marschall HU 2018 Role of bile acids in metabolic control. Trends in Endocrinology and Metabolism 29 31-41. (https://doi.org/10.1016/j.tem.2017.11.002)

Munukka E, Rintala A, Toivonen R, Nylund M, Yang B, Takanen A, Hanninen A, Vuopio J, Huovinen P, Jalkanen S, et al. 2017 Faecalibacterium prausnitzii treatment improves hepatic health and reduces adipose tissue inflammation in high-fat fed mice. ISME Journal 11 1667-1679. (https://doi.org/10.1038/ismej.2017.24)

Neyrinck AM, Possemiers S, Druart C, Van de Wiele T, De Backer F, Cani PD, Larondelle Y \& Delzenne NM 2011 Prebiotic effects of wheat arabinoxylan related to the increase in bifidobacteria, Roseburia and Bacteroides/Prevotella in diet-induced obese mice. PLOS ONE 6 e20944. (https://doi.org/10.1371/journal.pone.0020944)

Neyrinck AM, Possemiers S, Verstraete W, De Backer F, Cani PD \& Delzenne NM 2012a Dietary modulation of clostridial cluster XIVa gut bacteria (Roseburia spp.) by chitin-glucan fiber improves host metabolic alterations induced by high-fat diet in mice. Journal of Nutritional Biochemistry 23 51-59. (https://doi.org/10.1016/j. jnutbio.2010.10.008)

Neyrinck AM, Van Hee VF, Piront N, De Backer F, Toussaint O, Cani PD \& Delzenne NM 2012b Wheat-derived arabinoxylan oligosaccharides with prebiotic effect increase satietogenic gut peptides and reduce metabolic endotoxemia in diet-induced obese mice. Nutrition and Diabetes 2 e28. (https://doi.org/10.1038/nutd.2011.24)

Neyrinck AM, Van Hee VF, Bindels LB, De Backer F, Cani PD \& Delzenne NM 2013 Polyphenol-rich extract of pomegranate peel alleviates tissue inflammation and hypercholesterolaemia in high-fat diet-induced obese mice: potential implication of the gut microbiota. British Journal of Nutrition 109 802-809. (https://doi.org/10.1017/ S0007114512002206)

Nicolucci AC, Hume MP, Martinez I, Mayengbam S, Walter J \& Reimer RA 2017 Prebiotics reduce body fat and alter intestinal microbiota in children who are overweight or with obesity. Gastroenterology 153 711-722. (https://doi.org/10.1053/j.gastro.2017.05.055)

Nilsson AC, Johansson-Boll EV \& Bjorck IM 2015 Increased gut hormones and insulin sensitivity index following a 3-d intervention with a barley kernel-based product: a randomised cross-over study in healthy middle-aged subjects. British Journal of Nutrition 114 899-907. (https://doi.org/10.1017/S0007114515002524)

Olivares M, Rodriguez J, Potgens SA, Neyrinck AM, Cani PD, Bindels LB \& Delzenne NM 2019 The Janus face of cereals: wheat-derived prebiotics counteract the detrimental effect of gluten on metabolic homeostasis in mice fed a high-fat/high-sucrose diet. Molecular Nutrition and Food Research 63 e1900632. (https://doi.org/10.1002/mnfr.201900632)
Park SS, Lee YJ, Song S, Kim B, Kang H, Oh S \& Kim E 2018 Lactobacillus acidophilus NS1 attenuates diet-induced obesity and fatty liver. Journal of Endocrinology 237 87-100. (https://doi.org/10.1530/JOE-17-0592)

Plovier H, Everard A, Druart C, Depommier C, Van Hul M, Geurts L, Chilloux J, Ottman N, Duparc T, Lichtenstein L, et al. 2017 A purified membrane protein from Akkermansia muciniphila or the pasteurized bacterium improves metabolism in obese and diabetic mice. Nature Medicine 23 107-113. (https://doi.org/10.1038/nm.4236)

Potgens SA, Sboarina M \& Bindels LB 2018 Polyunsaturated fatty acids, polyphenols, amino acids, prebiotics: can they help to tackle cancer cachexia and related inflammation? Current Opinion in Clinical Nutrition and Metabolic Care 21 458-464. (https://doi.org/10.1097/ MCO.0000000000000505)

Qiao Y, Sun J, Xia S, Tang X, Shi Y \& Le G 2014 Effects of resveratrol on gut microbiota and fat storage in a mouse model with highfat-induced obesity. Food and Function 5 1241-1249. (https://doi. org/10.1039/c3fo60630a)

Qin J, Li Y, Cai Z, Li S, Zhu J, Zhang F, Liang S, Zhang W, Guan Y, Shen D, et al. 2012 A metagenome-wide association study of gut microbiota in type 2 diabetes. Nature 490 55-60. (https://doi.org/10.1038/ nature11450)

Rabot S, Membrez M, Bruneau A, Gerard P, Harach T, Moser M, Raymond F, Mansourian R \& Chou CJ 2010 Germ-free C57BL/6J mice are resistant to high-fat-diet-induced insulin resistance and have altered cholesterol metabolism. FASEB Journal 24 4948-4959. (https:// doi.org/10.1096/fj.10-164921)

Respondek F, Gerard P, Bossis M, Boschat L, Bruneau A, Rabot S, Wagner A \& Martin JC 2013 Short-chain fructo-oligosaccharides modulate intestinal microbiota and metabolic parameters of humanized gnotobiotic diet induced obesity mice. PLOS ONE 8 e71026. (https:// doi.org/10.1371/journal.pone.0071026)

Rodriguez J, Pierre N, Naslain D, Bontemps F, Ferreira D, Priem F, Deldicque L \& Francaux M 2017 Urolithin B, a newly identified regulator of skeletal muscle mass. Journal of Cachexia, Sarcopenia and Muscle 8 583-597. (https://doi.org/10.1002/jcsm.12190)

Rodriguez J, Hiel S \& Delzenne NM 2018 Metformin: old friend, new ways of action-implication of the gut microbiome? Current Opinion in Clinical Nutrition and Metabolic Care 21 294-301. (https://doi. org/10.1097/MCO.0000000000000468)

Rodriguez J, Hiel S, Neyrinck AM, Le Roy T, Potgens SA, Leyrolle Q, Pachikian BD, Gianfrancesco MA, Cani PD, Paquot N, et al. 2020 Discovery of the gut microbial signature driving the efficacy of prebiotic intervention in obese patients. Gut 69 1975-1987. (https:// doi.org/10.1136/gutjnl-2019-319726)

Ryu D, Mouchiroud L, Andreux PA, Katsyuba E, Moullan N, NicoletDit-Felix AA, Williams EG, Jha P, Lo Sasso G, Huzard D, et al. 2016 Urolithin A induces mitophagy and prolongs lifespan in C. elegans and increases muscle function in rodents. Nature Medicine $\mathbf{2 2}$ 879-888. (https://doi.org/10.1038/nm.4132)

Sarma SM, Singh DP, Singh P, Khare P, Mangal P, Singh S, Bijalwan V, Kaur J, Mantri S, Boparai RK, et al. 2018 Finger millet arabinoxylan protects mice from high-fat diet induced lipid derangements, inflammation, endotoxemia and gut bacterial dysbiosis. International Journal of Biological Macromolecules 106 994-1003. (https://doi. org/10.1016/j.ijbiomac.2017.08.100)

Schugar RC, Shih DM, Warrier M, Helsley RN, Burrows A, Ferguson D, Brown AL, Gromovsky AD, Heine M, Chatterjee A, et al. 2017 The TMAO-producing enzyme flavin-containing monooxygenase 3 regulates obesity and the beiging of white adipose tissue. Cell Reports 19 2451-2461. (https://doi.org/10.1016/j.celrep.2017.05.077)

Serino M, Luche E, Gres S, Baylac A, Berge M, Cenac C, Waget A, Klopp P, Iacovoni J, Klopp C, et al. 2012 Metabolic adaptation to a highfat diet is associated with a change in the gut microbiota. Gut $\mathbf{6 1}$ 543-553. (https://doi.org/10.1136/gutjnl-2011-301012)

Sheng Y, Liu J, Zheng S, Liang F, Luo Y, Huang K, Xu W \& He X 2019 Mulberry leaves ameliorate obesity through enhancing brown adipose https://joe.bioscientifica.com

https://doi.org/10.1530/JOE-20-0499 (c) 2021 Society for Endocrinology Published by Bioscientifica Ltd. Printed in Great Britain 
tissue activity and modulating gut microbiota. Food and Function 10 4771-4781. (https://doi.org/10.1039/c9fo00883g)

Suriano F, Bindels LB, Verspreet J, Courtin CM, Verbeke K, Cani PD, Neyrinck AM \& Delzenne NM 2017 Fat binding capacity and modulation of the gut microbiota both determine the effect of wheat bran fractions on adiposity. Scientific Reports 7 5621. (https://doi. org/10.1038/s41598-017-05698-y)

Udayappan S, Manneras-Holm L, Chaplin-Scott A, Belzer C, Herrema H, Dallinga-Thie GM, Duncan SH, Stroes ESG, Groen AK, Flint HJ, et al. 2016 Oral treatment with Eubacterium hallii improves insulin sensitivity in $\mathrm{db} / \mathrm{db}$ mice. NPJ Biofilms and Microbiomes 216009. (https://doi.org/10.1038/npjbiofilms.2016.9)

van der Beek CM, Canfora EE, Kip AM, Gorissen SHM, Olde Damink SWM, van Eijk HM, Holst JJ, Blaak EE, Dejong CHC \& Lenaerts K 2018 The prebiotic inulin improves substrate metabolism and promotes short-chain fatty acid production in overweight to obese men. Metabolism: Clinical and Experimental 87 25-35. (https:// doi.org/10.1016/j.metabol.2018.06.009)

Van Hul M, Geurts L, Plovier H, Druart C, Everard A, Stahlman M, Rhimi M, Chira K, Teissedre PL, Delzenne NM, et al. 2018 Reduced obesity, diabetes, and steatosis upon cinnamon and grape pomace are associated with changes in gut microbiota and markers of gut barrier. American Journal of Physiology: Endocrinology and Metabolism 314 E334-E352. (https://doi.org/10.1152/ajpendo.00107.2017)

Vrieze A, Van Nood E, Holleman F, Salojarvi J, Kootte RS, Bartelsman JF, Dallinga-Thie GM, Ackermans MT, Serlie MJ, Oozeer R, et al. 2012 Transfer of intestinal microbiota from lean donors increases insulin sensitivity in individuals with metabolic syndrome. Gastroenterology 143 913.e7-916.e7. (https://doi.org/10.1053/j.gastro.2012.06.031)

Wang J, Tang H, Zhang C, Zhao Y, Derrien M, Rocher E, van-Hylckama Vlieg JE, Strissel K, Zhao L, Obin M, et al. 2015 Modulation of gut microbiota during probiotic-mediated attenuation of metabolic syndrome in high fat diet-fed mice. ISME Journal 9 1-15. (https://doi. org/10.1038/ismej.2014.99)

Wang H, Hong T, Li N, Zang B \& Wu X 2018a Soluble dietary fiber improves energy homeostasis in obese mice by remodeling the gut microbiota. Biochemical and Biophysical Research Communications 498 146-151. (https://doi.org/10.1016/j.bbrc.2018.02.017)

Wang H, Zhang X, Wang S, Li H, Lu Z, Shi J \& Xu Z 2018b Mannanoligosaccharide modulates the obesity and gut microbiota in high-fat diet-fed mice. Food and Function 9 3916-3929. (https://doi. org/10.1039/c8fo00209f)
Wang P, Gao J, Ke W, Wang J, Li D, Liu R, Jia Y, Wang X, Chen X, Chen F, et al. 2020 Resveratrol reduces obesity in high-fat diet-fed mice via modulating the composition and metabolic function of the gut microbiota. Free Radical Biology and Medicine 156 83-98. (https://doi. org/10.1016/j.freeradbiomed.2020.04.013)

Weitkunat K, Stuhlmann C, Postel A, Rumberger S, Fankhanel M, Woting A, Petzke KJ, Gohlke S, Schulz TJ, Blaut M, et al. 2017 Shortchain fatty acids and inulin, but not guar gum, prevent diet-induced obesity and insulin resistance through differential mechanisms in mice. Scientific Reports 7 6109. (https://doi.org/10.1038/s41598-017-06447-x)

Wu H, Tremaroli V, Schmidt C, Lundqvist A, Olsson LM, Kramer M, Gummesson A, Perkins R, Bergstrom G \& Backhed F 2020 The gut microbiota in prediabetes and diabetes: a population-based crosssectional study. Cell Metabolism 32 379.e3-390.e3. (https://doi. org/10.1016/j.cmet.2020.06.011)

Xia B, Shi XC, Xie BC, Zhu MQ, Chen Y, Chu XY, Cai GH, Liu M, Yang SZ, Mitchell GA, et al. 2020 Urolithin A exerts antiobesity effects through enhancing adipose tissue thermogenesis in mice. PLoS Biology 18 e3000688. (https://doi.org/10.1371/journal. pbio.3000688)

Yamashita H, Maruta H, Jozuka M, Kimura R, Iwabuchi H, Yamato M, Saito T, Fujisawa K, Takahashi Y, Kimoto M, et al. 2009 Effects of acetate on lipid metabolism in muscles and adipose tissues of type 2 diabetic Otsuka Long-Evans Tokushima Fatty (OLETF) rats. Bioscience, Biotechnology, and Biochemistry 73 570-576. (https://doi.org/10.1271/ bbb.80634)

Zhang X, Shen D, Fang Z, Jie Z, Qiu X, Zhang C, Chen Y \& Ji L 2013 Human gut microbiota changes reveal the progression of glucose intolerance. PLoS ONE 8 e71108. (https://doi.org/10.1371/journal. pone.0071108)

Zhao L, Zhang F, Ding X, Wu G, Lam YY, Wang X, Fu H, Xue X, Lu C, Ma J, et al. 2018 Gut bacteria selectively promoted by dietary fibers alleviate type 2 diabetes. Science 359 1151-1156. (https://doi. org/10.1126/science.aao5774)

Zhou J, Martin RJ, Raggio AM, Shen L, McCutcheon K \& Keenan MJ 2015 The importance of GLP-1 and PYY in resistant starch's effect on body fat in mice. Molecular Nutrition and Food Research 59 1000-1003. (https://doi.org/10.1002/mnfr.201400904)

Zhou L, Xiao X, Zhang Q, Zheng J \& Deng M 2019 Deciphering the anti-obesity benefits of resveratrol: the 'gut microbiota-adipose tissue' axis. Frontiers in Endocrinology 10 413. (https://doi.org/10.3389/ fendo.2019.00413)

Received in final form 10 December 2020

Accepted 25 January 2021

Accepted Manuscript published online 28 January 2021 (c) 2021 Society for Endocrinology Published by Bioscientifica Ltd. Printed in Great Britain 